ARTICLE

\title{
The p63 C-terminus is essential for murine oocyte integrity
}

\author{
Anna Maria Lena (1) 1, Valerio Rossi ${ }^{2}$, Susanne Osterburg ${ }^{3}$, Artem Smirnov (1) 1,8, Christian Osterburg (1) ${ }^{3}$, \\ Marcel Tuppi ${ }^{3,9}$, Angela Cappello ${ }^{1}$, Ivano Amelio (1) 1,4, Volker Dötsch (1) ${ }^{3}$, Massimo De Felici², \\ Francesca Gioia Klinger (10 2, Margherita Annicchiarico-Petruzzelli ${ }^{5}$, Herbert Valensise ${ }^{6,7}$, Gerry Melino (i) ${ }^{1 凶} \&$ \\ Eleonora Candi (iD) $1,5 \bowtie$
}

The transcription factor p63 mediates distinct cellular responses, primarily regulating epithelial and oocyte biology. In addition to the two amino terminal isoforms, TAp63 and $\Delta N p 63$, the 3 '-end of p63 mRNA undergoes tissue-specific alternative splicing that leads to several isoforms, including $p 63 \alpha, p 63 \beta$ and $p 63 \gamma$. To investigate in vivo how the different isoforms fulfil distinct functions at the cellular and developmental levels, we developed a mouse model replacing the p63 $\alpha$ with p63 $\beta$ by deletion of exon 13 in the Trp63 gene. Here, we report that whereas in two organs physiologically expressing $p 63 \alpha$, such as thymus and skin, no abnormalities are detected, total infertility is evident in heterozygous female mice. A sharp reduction in the number of primary oocytes during the first week after birth occurs as a consequence of the enhanced expression of the pro-apoptotic transcriptional targets Puma and Noxa by the tetrameric, constitutively active, TAp63 $\beta$ isoform. Hence, these mice show a condition of ovary dysfunction, resembling human primary ovary insufficiency. Our results show that the p63 C-terminus is essential in TAp63 $\alpha$-expressing primary oocytes to control cell death in vivo, expanding the current understanding of human primary ovarian insufficiency.

\footnotetext{
${ }^{1}$ Department of Experimental Medicine, University of Rome "Tor Vergata", Rome, Italy. ${ }^{2}$ Department of Biomedicine and Prevention, University of Rome Tor Vergata, Rome, Italy. ${ }^{3}$ Institute of Biophysical Chemistry, Center for Biomolecular Magnetic Resonance and Cluster of Excellence Macromolecular Complexes (CEF), Goethe University, Frankfurt, Germany. ${ }^{4}$ School of Life Sciences, University of Nottingham, Nottingham, UK. ${ }^{5}$ IDI-IRCCS, Via dei Monti di Creta, Rome, Italy. ${ }^{6}$ Department of Surgery, University of Rome "Tor Vergata", Rome, Italy. ${ }^{7}$ Policlinico "Casilino", Rome, Italy. ${ }^{8}$ Present address: Ludwig Institute for Cancer Research, University of Oxford, Oxford OX3 7DQ, UK. ${ }^{9}$ Present address: The Francis Crick Institute, London NW11ST, UK.

凶email: melino@uniroma2.it; candi@uniroma2.it
} 
T he transcription factor p63 is crucial for mouse embryo cranio-facial, skin, and limb development ${ }^{1-3}$. The TP63 gene harbours two different promoters, resulting in two $\mathrm{N}$-terminal isoforms (TAp63 and $\Delta \mathrm{Np} 63$ ). The full-length TAp63 isoform has an N-terminal transactivation domain (TAD), while the N-terminal truncated $\Delta \mathrm{Np} 63$ variant lacks this region but still shows transcriptional activity on certain promoters ${ }^{4,5}$. The $\Delta \mathrm{Np} 63 \mathrm{a}$ isoform is mainly expressed in ectodermal and endodermal-derived tissues, including the epidermis, skin appendages, some simple epithelia, and the thymus $^{4,6,7}$. In contrast, the TAp63a isoform is present both in the male and female germlines of reproductive organs, in adult muscle tissues and in cardiac muscle precursors ${ }^{8,9}$. The in vivo contributions of $\mathrm{p} 63 \mathrm{~N}$-terminal variants to embryonic development are clearly evident upon selective $\Delta \mathrm{Np} 63$ and TAp63 knockout $(\mathrm{KO})$ mice ${ }^{8,10}$ : striking developmental abnormalities have been observed in $\Delta \mathrm{Np} 63$-null mice ${ }^{11}$, in which the indispensable role of the $\Delta \mathrm{Np} 63$ isoform in epithelial biology has been genetically demonstrated. On the other hand, TAp63 KO mice show normal development ${ }^{8,12}$ with abnormalities in cellular senescence that prevent premature tissue ageing and defects in glucose and lipid metabolism ${ }^{10}$. TAp63 is constitutively expressed in primary oocytes arrested at the dyctiate stage of the meiotic prophase I within the primordial/primary follicles ${ }^{8,9}$. Its activation is essential to eliminate such oocytes suffering DNAdamage-induced death and in this way to maintain the integrity of the female germline; such monitoring plays a crucial role in the quality control of the female ovarian reserve ${ }^{8,13,14}$. Although TAp63 and yet another, N-terminally elongated isoform, GTAp63a 15,16 , has also been detected in male germ cells, its role in this context has not yet been fully clarified ${ }^{17-19}$.

Additionally, p63 can be expressed as several C-terminal isoforms, including p63a, $\mathrm{p} 63 \beta$, and $\mathrm{p} 63 \gamma^{3}$. Whereas the functional in vivo roles of p63 $\mathrm{N}$-terminal variants have been relatively well studied, the in vivo functions of $\mathrm{p} 63 \mathrm{C}$-terminal variants have not yet been investigated. Indeed, by alternative splicing, at least three different C-terminal isoforms, p63a, p63 $\beta$, and p $63 \gamma$, are obtained in a tissue-specific manner, both for the TAp63 and $\Delta \mathrm{Np} 63$ isoforms ${ }^{4}$. Only the p63a variant harbours the sterile alpha motif (SAM) domain, which is a proposed protein-protein interaction domain of still unknown function ${ }^{4,20,21}$, and the transactivation inhibitory domain (TID), which is involved in transcriptional inhibition $^{22,23}$. Structural and biochemical studies have demonstrated that the p63a C-terminus contains multiple regulatory elements with different functions undergoing post-translational modifications ${ }^{24-26}$. For instance, the TID is important for the formation of the autoinhibitory dimeric complex of TAp63a in primary oocytes. The inactive dimeric state is thereby stabilized by an interaction network between the N-terminal transactivation domain (TAD) and the TID ${ }^{24,27}$. After detection of DNA damage in the oocytes, TAp63a gets phosphorylated by the priming kinase Chk2 and the executioner kinase CK1, which results in disruption of the dimer and allows formation of the active tetramer ${ }^{28,29}$. Subsequently, active TAp63a orchestrates oocyte death by inducing apoptosis via the target genes p53-upregulatedmodulator-of-apoptosis (Puma) and phorbol-12-myristate-13acetate-induced protein-1 (PMAIP1, herinafter Noxa) ${ }^{13,29,30}$.

Heterozygous mutations in the p63 gene are the cause of five human developmental disorders. The ectrodactyly, ectodermal dysplasia, and cleft lip/palate syndrome (EEC, Online Mendelian Inheritance in Man, OMIM 604292), is mainly characterized by ectrodactyly, ectodermal dysplasia, limb defects and cleft lip and palate (CLP), while the limb-mammary syndrome (LMS, OMIM603543) is defined by split-hand-feet malformations, cleft palate (CP), mammary-gland hypoplasia and/or nipple aplasia. Acro-dermato-ungual-lacrimal-tooth syndrome (ADULT,
OMIM103285) patients show limb malformations, finger- and toenail dysplasia, hypoplastic breasts and nipples, primary hypodontia, and loss of permanent teeth. These three syndromes can be summarized as ELA (EEC, LMS, ADULT) syndrome due to overlapping of the patient's manifestations making classification in single syndromes difficult ${ }^{31-33}$. In contrast to the already mentioned syndromes, isolated split-hand/foot malformation (SHFM4, OMIM605289) only affects the limb development. Ankyloblepharon-ectodermal defects-cleft lip/palate (AEC, OMIM 106260) and Rapp-Hodgkin syndrome (RHS, OMIM129400) also show overlapping clinical features and are suggested to represent the same entity (in the following AEC/ RHS). Both syndromes are characterized by CLP and ectodermal dysplasia ${ }^{34}$.

For each of the two syndromic entities, the mutations cluster in specific domains of p63, indicating a genotype-to-phenotype correlation. ELA mutations are mainly located in the DBD, suggested to inhibit DNA binding and transactivation or in the Cterminus $^{35}$, whereas AEC/RHS mutations are only present in the C-terminus, inducing aggregation and, thus, inactivation of the protein $^{36}$.

Previous work correlated TP63 mutations to female fertility. In case of a heterozygous two nucleotides deletion (delTT1576) ${ }^{37}$, the ovary is completely absent leading to infertility. Furthermore, there are two related, female AEC/RHS patients (delC1783) ${ }^{38}$ described suffering from premature menopause around the age of 30 years. Recently, Tucker et al. described TP63 defects as the cause of isolated primary ovarian insufficiency (POI). Via introduction of nonsense mutations in the C-terminal part of the SAM domain (R555*, W559*) ${ }^{39,40}$, p63a is missing the TID. However, the underlying molecular mechanisms require further elucidation.

To investigate the global functions of the p63 variants and to understand the p63 C-terminus contribution, we generate genetically modified mice in which exon 13 was deleted $(\Delta 13)$ to replace p63a with p63 $\beta$. Heterozygous female mice bearing wildtype (WT) and exon 13-deleted (HET $\Delta 13$ p63) alleles are sterile due to the rapid loss of the primary oocytes stockpile. Our results indicate that the p63 C-terminus plays different roles in TAp63 and $\Delta \mathrm{Np} 63$ isoforms and that the TID inhibitory effect is required in TAp63a-expressing tissues to control cell death. In the four p63 mutations already described to affect female fertility, the TID is missing due to early stop codons in the SAM or insertion/deletion of DNA bp creating another reading frame. Here, we report the likely mechanism for these mutations leading to infertility of the female patients. Furthermore, we investigate the transactivation and oligomeric conformation of other syndromic TAp63a mutants enabling predictions of the patients' oocyte fate. Based on our experiments, we can conclude that female HET $\Delta 13$ p63 mice represent an animal model for POI.

\section{Results}

Generation of the $\Delta 13 \mathrm{p} 63$ heterozygous mice. To investigate the roles of p63 isoforms in vivo, we decided to selectively delete Trp63 exon 13. Exon 13 encodes the SAM domain, typical of the p63a isoform, and its genomic deletion leads to expression of the p63 $\beta$ isoform instead of the p63 $\alpha$ isoform, as shown in detail by nucleotide and amino acid sequence alignments (Supplementary Fig. 1a, b). We generated an exon 13 floxed allele introducing, by homologous recombination, two loxP sites into Trp63 introns flanking the exon $133^{\prime}$ and 5' ends (Fig. 1a). Heterozygous floxed allele mice were then crossed with CMV-Cre mice to obtain the heterozygous $\Delta 13$ p63 strain (HET $\Delta 13 p 63$, indicated as HET in figures, Fig. 1b-d). HET $\Delta 13$ p63 mice were born alive at the expected Mendelian ratios (Fig. 1b) and showed normal development and morphology both at birth and in 
a

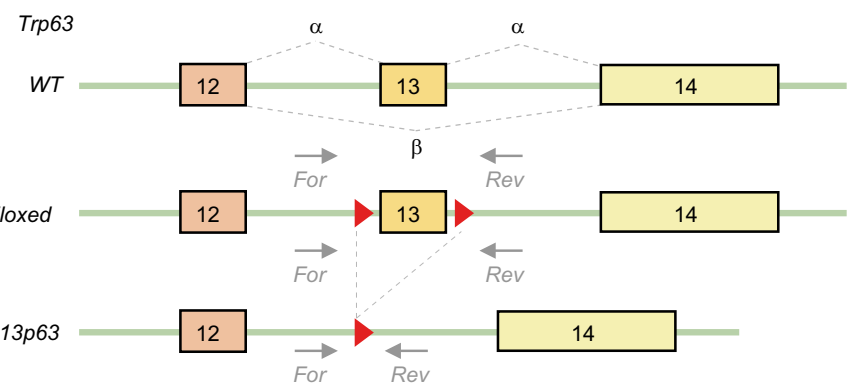

C

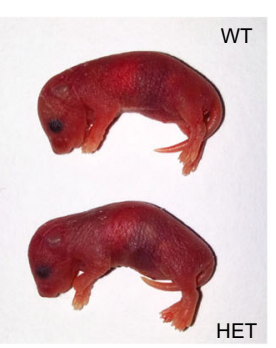

d

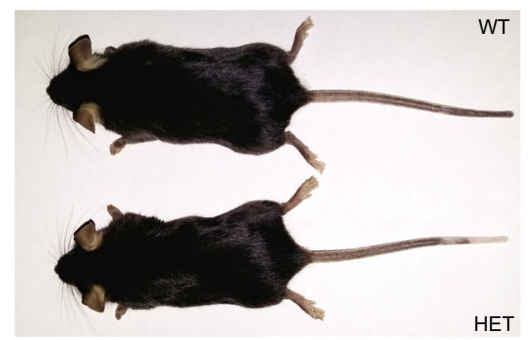

b

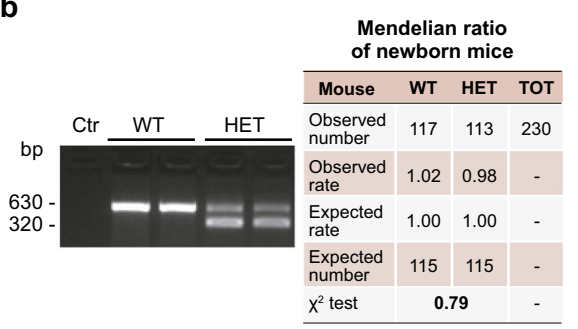

e

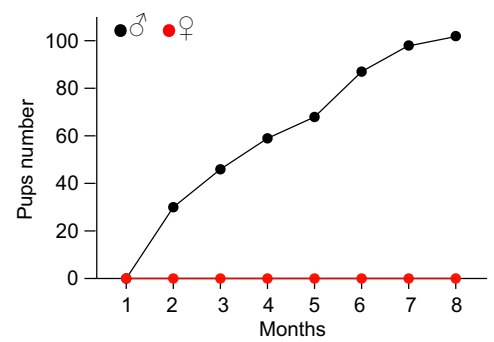

h
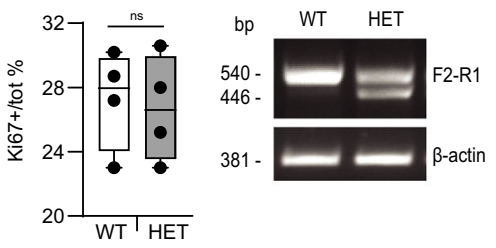

i
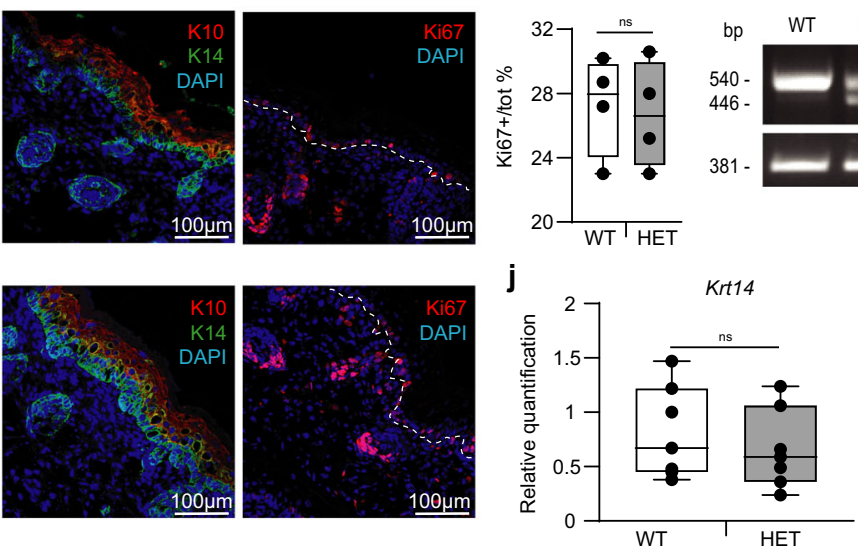

k

I

m

n $\mathrm{kDa}$

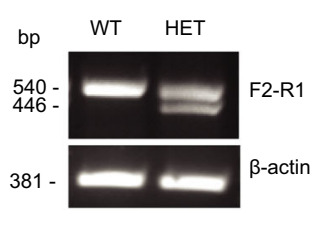

$\underline{100 \mu m}$

o

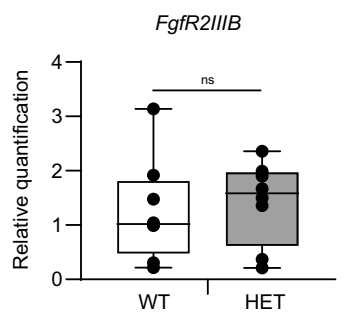

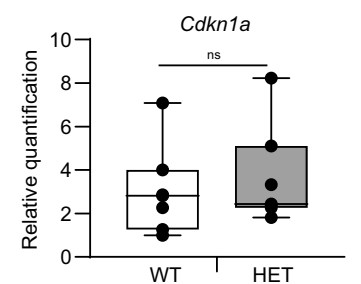

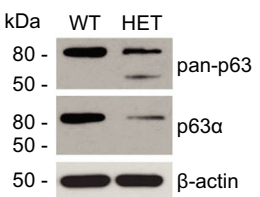

HET
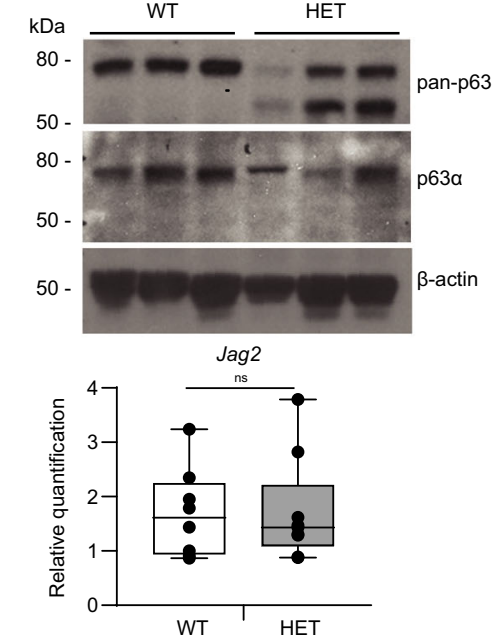

adulthood compared to wild-type (WT) mice (Fig. 1c, d). To verify that both $\mathrm{p} 63 \alpha$ and $\mathrm{p} 63 \beta$ isoforms were expressed in HET $\Delta 13$ p63 animals, we performed semi-quantitative PCR using cDNA derived from RNA extracted from newborn epidermis and thymus samples as a template. Exons 11, 12, and 13 were amplified using forward primers (F1, F2, and F3) and a reverse primer (R1) in the Trp63 3'UTR region (see Supplementary
Fig. 1a-d) to show that in p63 HET $\Delta 13$ p63 animals, only exon 13 was missing in one of the two alleles. The expression of the p63 $\gamma$ isoform was unaltered in both WT and p63 HET $\Delta 13$ p63 animals (primers F4-R2, Supplementary Fig. 1d). Sequencing of the PCR fragment after gel extraction demonstrated the correct splicing leading to p63 $\beta$ mRNA (Supplementary Fig. 1c). Unfortunately, HET $\Delta 13$ p63 females mated with both HET 
Fig. 1 Generation and characterization of $\Delta \mathbf{1 3 p 6 3}$ heterozygous mice. a Strategy for the generation of $\Delta 13 p 63$ heterozygous mice (HET $\Delta 13 p 63$, indicated as HET in figure). The targeting vector was generated by inserting loxP sites (red triangles) into the genomic regions flanking exon 13 to obtain a floxed allele. Heterozygous floxed mice were crossed with CMV-Cre transgenic mice to obtain HET mice. The primers used for genotyping the WT and HET mice are shown (For, forward; Rev, reverse); b Agarose gel electrophoresis of the PCR products obtained using genomic DNA from WT and HET mice as a template. Ctr indicates a no-template PCR. Table on the right side shows Mendelian distribution of genotypes in newborn mice (WT and HET). $p>0.05$ by $\mathrm{X}^{2}$ test; $\mathbf{c}$ Newborn WT and HET male mice; $\mathbf{d}$ Three-month-old WT and HET male mice; e Pups number obtained by crossing HET males (black line, $n=4$ ) and HET females (red line, $n=10$ ) with WT mice for a period of 8 months; $\mathbf{f}$ Hematoxylin and eosin staining of P1 backskin sections of WT and HET mice; g IF staining of K10/K14 and Ki67 marker on P1 backskin sections of WT and HET. Ki67 positive nuclei counting was performed on P1 backskin sections of WT $(n=6)$ and HET $(n=6)$ mice. Epidermis Ki67 positive nuclei percentage was shown in the graph on the right side. The centre of the boxplots represents median, boxes represent first (25\%) and third (75\%) quartiles, whiskers extend to the most extreme datapoints that are no more than 1.5 -fold of the interquartile range from the box. Single values are plotted as individual points. $p$-value $>0.05$ by two-tailed unpaired Student's $t$-test; $\mathbf{h}$ Semiquantitative PCR analysis of p63 isoforms expression in P1 epidermises of WT and HET mice; $\mathbf{i}$ WB analysis of p63 isoforms expression in WT and HET mice protein extracts of cultured primary keratinocytes. $\beta$-actin was used as loading control. $\mathbf{j}$ Boxplots showing Krt14 and Cdkn1a (p21) expression quantification by RT-qPCR in epidermises of WT $(n=7)$ and HET $(n=7)$ mice. $p$-value $>0.05$ by two-tailed unpaired Student's $t$-test; $\mathbf{k}$ Haematoxylin and eosin staining of P1 thymus sections of WT and HET mice; I IF staining of K5/K8 markers on thymus sections of WT and HET mice; $\mathbf{m}$ Semi-quantitative PCR analysis of p63 isoforms expression in P1 thymus of WT and HET mice; $\mathbf{n}$ WB analysis of p63 isoforms expression in protein extracts of WT and HET mice P1 thymuses. $\beta$-actin was used as loading control. o Boxplots showing FgfR2Illb and Jag2 expression quantification by RT-qPCR in thymuses of WT $(n=7)$ and HET $(n=7)$ mice. $p$-value $>0.05$ by two-tailed unpaired Student's $t$-test. The images shown are representative of all the experiments performed (at least $n=6$ ) Source data are provided as a Source Data file.

$\Delta 13$ p63 and WT males never became pregnant over a period of 8 months, excluding the possibility of obtaining $\Delta 13$ p63 homozygous animals (Fig. 1e). HET $\Delta 13$ p63 males were fertile, and when crossed with WT females, they generated litters with normal numbers and Mendelian genotype ratios (Fig. 1c), indicating that the HET $\Delta 13$ p63 female mice have selectively impaired fertility.

$\Delta 13$ p63 heterozygous mice develop normal skin and normal thymus. To understand the role of p63a in $\Delta$ Np63-expressing tissues, we evaluated the skin and thymus from newborn mice, two organs in which $\Delta \mathrm{Np} 63 \mathrm{a}$ is expressed at high levels and plays a functionally relevant role $6,7,41,42$. Histological characterization by haematoxylin-eosin (H/E) staining showed normal structures of the dorsal epidermis (Fig. 1f). The expression of the basal layer marker keratin (K) 14 (K14) and that of the differentiation layer marker K10 were identical between WT and HET $\Delta 13 \mathrm{p} 63$ mice, as was the expression of the proliferation marker Ki67 (Fig. 1g), suggesting normal proliferation and differentiation programmes in the HET $\Delta 13$ p63 epidermis. In the epidermis, the isoforms $(\Delta \mathrm{Np} 63 \alpha$ and $\Delta \mathrm{Np} 63 \beta)$ were expressed at 1:1 ratio at the mRNA level (Fig. 1h, Supplementary Fig. 1d). Western blot analysis also showed their expression, as indicated by staining of epidermal extracts with an anti-pan-p63 antibody (Fig. 1i). Furthermore, no differences were detected by Real Time (RT)-qPCR in the expression of two bona fide $\Delta \mathrm{Np} 63 a$ skin-specific target genes,

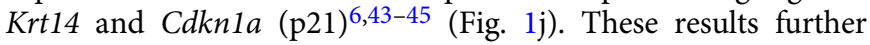
confirmed that the simultaneous expression of $\triangle \mathrm{Np} 63 \alpha$ and $\Delta N p 63 \beta$ in the HET $\Delta 13$ p63 epidermis does not affect normal function during development, possibly because the two variants act synergistically to transcribe the same target genes.

Similar results were obtained in the thymus, in which $\Delta \mathrm{Np} 63 a$, expressed in thymic epithelial cells (TECs), sustains thymus development through the regulation of two target genes, $\mathrm{FgfR} 2 \mathrm{IIIb}$ and Jag2 $2^{41,46-48}$. Histological characterization by haematoxylin-eosin staining showed normal organ structure (Fig. 1k). No differences were detected in the expression of the TEC differentiation markers K8 and K5 (Fig. 11). Both isoforms $(\triangle \mathrm{Np} 63 \alpha$ and $\Delta \mathrm{Np} 63 \beta)$ were detected in the thymus at the mRNA level (Fig. 1m, Supplementary Fig. 1d) and at the protein level (Fig. 1n), as indicated in the Western blot obtained using the anti-pan-p63 antibody. Additionally, no differences were detected by RT-qPCR in the expression of two bona fide thymus-specific $\Delta \mathrm{Np} 63 \mathrm{a}$ target genes, FgfR2IIIb and Jag2 ${ }^{41}$ (Fig. 10). Taken together, these data indicated that in $\Delta \mathrm{Np} 63$-expressing organs, the thymus and epidermis, the simultaneous expression of $\Delta \mathrm{Np} 63 \alpha$ and $\Delta \mathrm{Np} 63 \beta$ did not alter the organ development or the expression of p63 tissue-specific target genes.

$\Delta 13 p 63$ heterozygous females show primary ovary insufficiency. TAp63 isoforms are expressed both in male and female germlines $8,15,16$. During mouse ovary development, TAp63a starts to be expressed in primary oocytes around embryonic day 17 during the prophase of the first meiotic division ${ }^{8}$. At 4-7 days post-partum (dpp), all oocytes arrested at the dyctiate stage of the meiosis within primordial/primary follicles are TAp63a positive ${ }^{8}$. TAp63 is dispensable for ovarian development, as shown in TAp63 KO mice ${ }^{12}$; nevertheless, it plays a crucial role in the quality control of the primary oocytes being activated and inducing apoptosis in those presenting DNA damage ${ }^{8,13,14}$. Therefore, to understand the infertility phenotype observed in the heterozygous females, a deeper analysis of the ovarian tissues was carried out. Macroscopic analysis of postnatal day 45 (P45) ovaries from WT and HET $\Delta 13 \mathrm{p} 63$ females revealed evident organ size difference: postnatal day 45 ovaries from HET $\Delta 13$ p63 mice were significantly smaller than those from WT mice (Fig. 2a). Through semi-quantitative RT-qPCR analysis of TAp63 expression in ovaries from WT and HET $\triangle 13$ p63 females from day 17.5 of embryonic development (E17.5) to postnatal days 1 (P1), 3 (P3), 7 (P7), and 10 (P10), we confirmed that in WT mice TAp63a isoform is detectable at E17.5 and that its expression remained high in primordial and primary follicles (P1-P10; Fig. $2 b$ ). On the other hand, in HET $\Delta 13$ p63 ovaries, while there was the expected increase from E17.5 to P1 (Fig. 2b), at later time points, both p $63 \alpha$ and p $63 \beta$ mRNA levels decreased along with a reduction in oocytes number (Fig. $2 \mathrm{~d}-\mathrm{g}$ ), which appeared associated to increased apoptosis (Fig. 3). Since within the ovaries, p63 is exclusively expressed in the oocytes, depauperation of these population resulted in reduction of p63 mRNA. In HET $\Delta 13$ p63 ovaries, p63 $\gamma$ isoform and p53 mRNAs followed similar trend (Supplementary Fig. 1e). Western blot analysis showed that only the TAp63a isoform was detectable at very low levels in P1 HET $\Delta 13$ p63 ovary extracts, while the levels of the TAp63 $\beta$ variant were not appreciable (Fig. 2c). Mechanistically, however, we also proved that active TAp63 $\beta$ variant undergoes a high proteosomal degradation rate (Supplementary Fig. $2 \mathrm{a}-\mathrm{c}$ ). Thus, both oocytes depauperation and poor protein stability underlyed low expression level of TAp63 $\beta$ in HET $\Delta 13$ p63 ovary extracts. Histological 
a
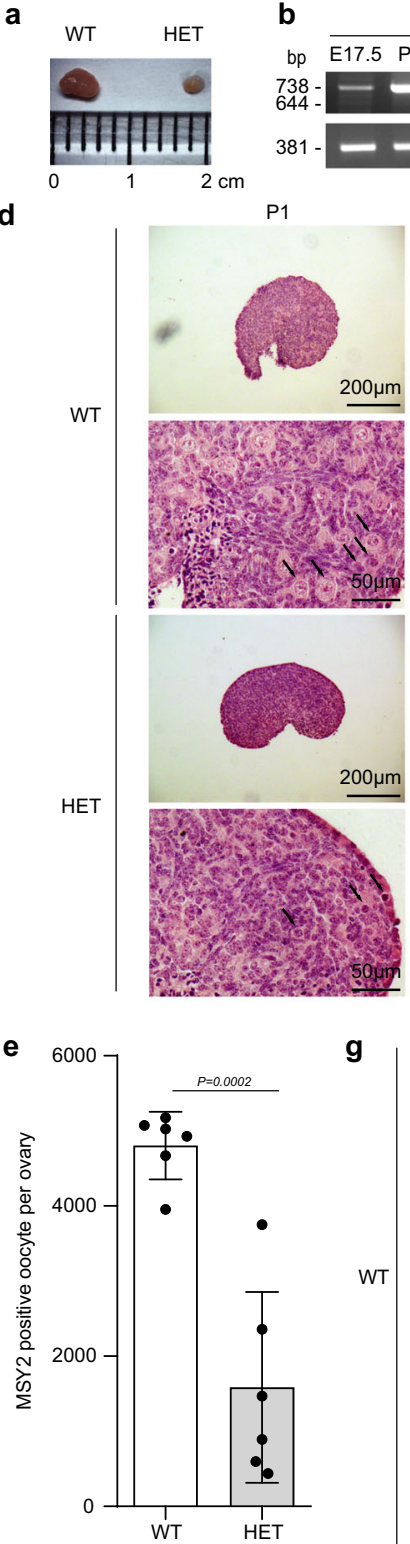

$\mathbf{f}$
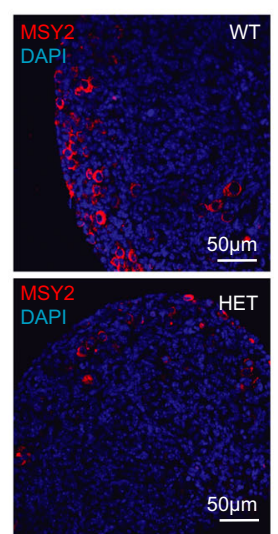

HET b

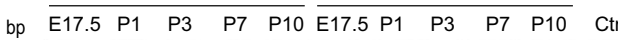
648

381

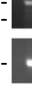

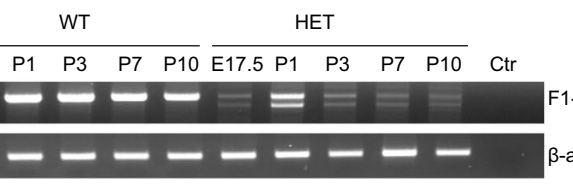

C

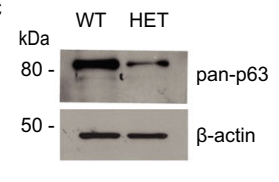

P5

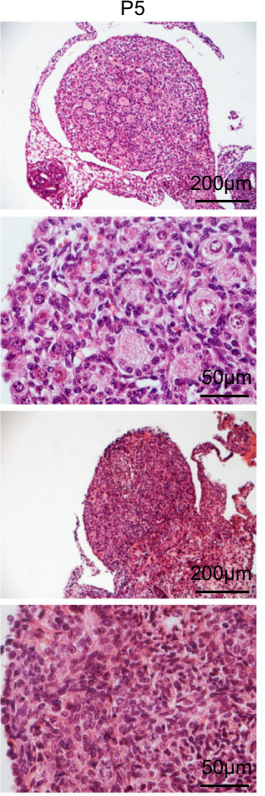

P1
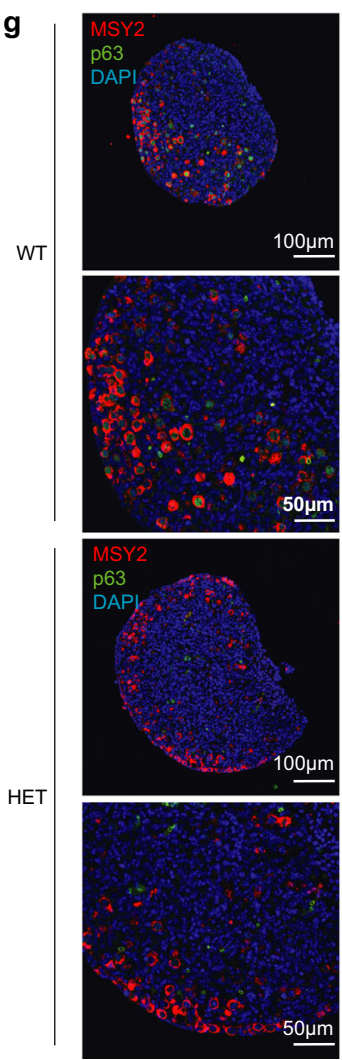

P10

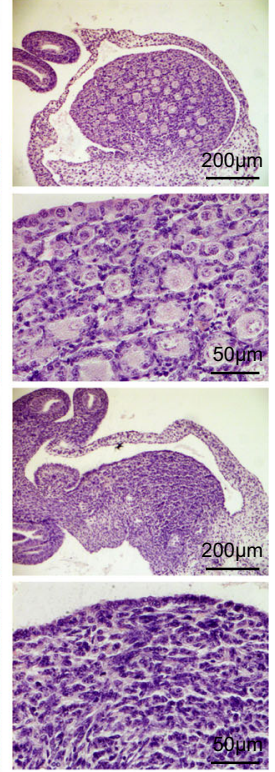

P5

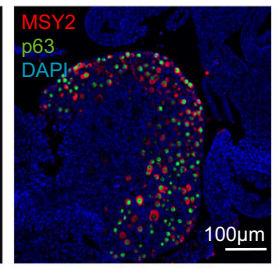

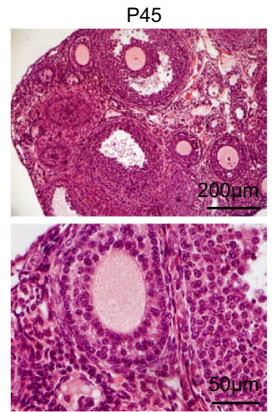
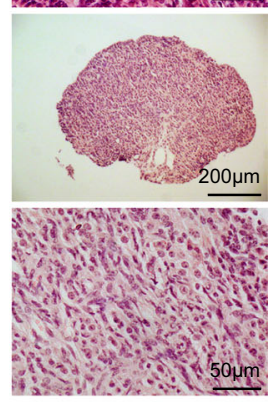

P10
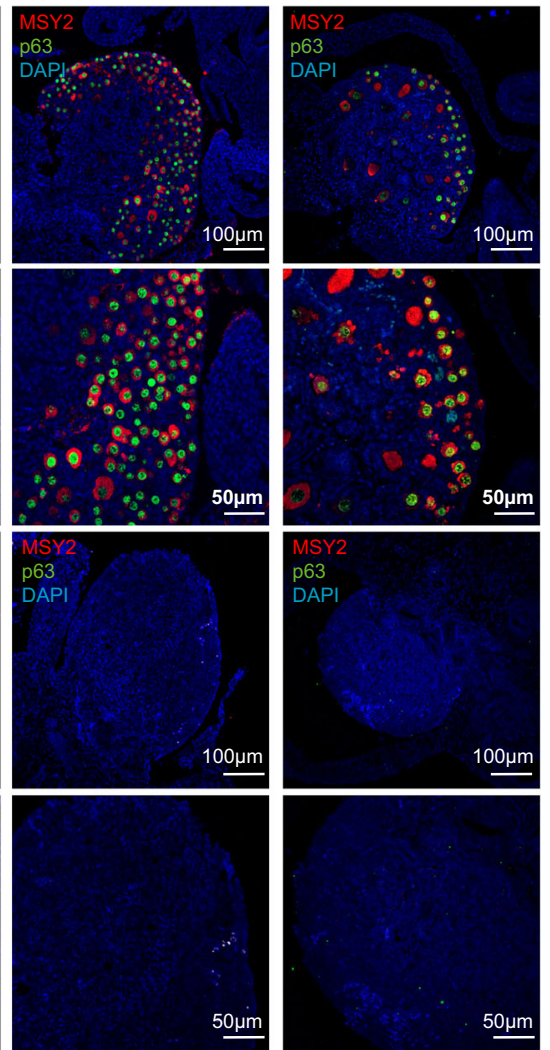

characterization by $\mathrm{H} / \mathrm{E}$ staining of $\mathrm{P} 1, \mathrm{P} 5, \mathrm{P} 10$, and $\mathrm{P} 45$ ovaries revealed that, while WT and HET $\Delta 13$ p63 P1 ovaries showed similar morphologies with the presence of primordial follicles (Fig. 2d, arrows), HET $\Delta 13$ p63 P5 and P10 ovaries presented an atretic morphology and almost total absence of follicles, aspects even more evident in HET $\Delta 13$ p63 P45 ovaries (Fig. 2d). Moreover, HET $\triangle 13$ p63 P1 ovaries showed significant less (40\%) numbers of oocytes identified by MSY2 compared to WT P1 ovaries (Fig. 2e, f). Double IF co-staining of MSY2 and p63 showed that in WT ovaries oocytes were positive for both as expected, while in HET $\Delta 13$ p63 ovaries, oocytes were mainly stained for MSY2 (Fig. 2g). These observations strongly support the hypothesis that oocytes depletion in heterozygous ovaries is correlated with the activity of TAp63 $\beta$ isoform, that is known to 
Fig. 2 Characterization of $\Delta \mathbf{1 3 p 6 3}$ heterozygous mouse ovaries. a P45 WT and HET $\Delta 13 p 63$ (indicated as HET in figure) ovary size; b Semi-quantitative PCR analysis of $p 63 \alpha$ and p63 $\beta$ isoforms expression E17.5, P1, P3, P7, and P10 ovaries of WT and HET mice. $\beta$-actin was used as housekeeping gene for normalization. Ctr indicates a no-template PCR; c WB analysis of p63 isoforms expression in protein extracts of P1 ovaries of WT $(n=3)$ and HET ( $n=3$ ) mice. $\beta$-actin was used as a loading control; $\mathbf{d}$ Haematoxylin and eosin staining at P1, P5, P10, and P45 (1, 5, 10, and 45 day post-partum, P) ovary sections of WT and HET mice. The panels on the right side are magnifications of areas of the panels on the left side. Arrows indicate oocytes; e MSY2 positive cell count/ovary; WT $(n=6)$ and HET $(n=6)$ ovaries. Data are presented as mean \pm SD, $p$-value by two-tailed unpaired Student's $t$-test; $f$ Oocytes IF staining for MSY2 (red) in P1 ovary sections of WT and HET mice; $\mathbf{g}$ MSY2 and p63 co-staining of P1, P5, and P10 ovary sections of WT and HET mice. The panels on the right are magnifications of areas of the panels on the left side. The images shown are representative of all the experiments performed (at least $n=$ 6) Source data are provided as a Source Data file.

be rapidly degraded (Supplementary Fig. 2a-c) ${ }^{23}$. Since TAp63 has also been detected in testis ${ }^{18}$, we compared P45 WT and HET $\Delta 13$ p63 testis. No abnormalities were observed at the gross morphology level. Morphology, as evaluated by PAS staining, did not reveal differences between WT and HET $\triangle 13 p 63$ testis (Supplementary Fig. 3a, b). Both TAp63 $\alpha$ and TAp63 $\beta$ mRNA variants were expressed at comparable levels (Supplementary Fig. 3c). Taken together, these results demonstrate that the simultaneous expression of TAp63 $\alpha$ and TAp63 $\beta$ causes a condition of ovary disfunction resembling primary ovary insufficiency (POI) in human females but does not affect testis morphology or function.

\section{$\Delta 13$ p63 heterozygous primary oocytes undergo uncontrolled} apoptotic death. To investigate in vivo the underlying molecular events driving HET $\Delta 13$ p63 infertility, we crossed HET $\Delta 13 p 63$ males with transgenic $\mathrm{p} 70$-c-Kit/GFP females ${ }^{49}$ to obtain p70-c$\mathrm{Kit} / \mathrm{GFP} / \Delta 13 \mathrm{p} 63$ heterozygous mice (also named WT-GFP or HET $\Delta 13$ p63-GFP mice). These mice showed green fluorescence only in primary oocytes, enabling us to follow their primary oocyte postnatal development ex vivo from P1 to P7. WT and HET $\triangle 13$ p63-GFP ovaries were isolated and cultured ex vivo, as shown in Fig. 3a. Confocal scanning of whole ovaries with $\mathrm{z}$ stacks acquisition every $5 \mu \mathrm{m}$ confirmed that under ex vivo conditions, apoptotic death occurs in a constitutive manner during primary follicle development without inducing damage (Fig. 3a). HET $\triangle 13$ p63-GFP ovaries, compared with WT ovaries, progressively and rapidly lost green fluorescent oocytes from P1 to P7; at day 7, the oocytes became undetectable (Fig. 3a). These striking results suggest that the oocytes disappeared due to an accelerated uncontrolled cell death programme mediated by TAp63 $\beta$. To test whether the primary oocytes died by overt apoptosis, we added the pan-caspase inhibitor Z-VAD to the HET $\triangle 13$ p63-GFP ovary culture medium. Since, distinguishing individual oocytes immediately after birth (P0-P3) is quite challenging, making virtually unreliable counts, we quantified the intensity of GFP fluorescence to estimate the number of oocytes in different conditions. Indeed, intensity of GFP is proportional to the number of oocytes, as assessed by manual counting of oocytes at P4 where they are clearly separated individually. After $\mathrm{Z}$-VAD treatment, we observed a significant delay in cell death, as indicated by the rescue of the green fluorescent oocytes in $\mathrm{Z}$ VAD-treated HET $\triangle 13$ p63-GFP ovaries compared to control DMSO-treated HET $\triangle 13$ p63-GFP ovaries (Fig. 3b, c). Accordingly, we observed significant upregulation of apoptotic TAp63 target genes involved in primordial follicle cell death, namely, Puma and Noxa, in HET $\Delta 13$ p63 compared to WT ovary samples (Fig. $3 \mathrm{~d}$ ). These results suggest that the expression of TAp63 $\beta$ is sufficient to trigger uncontrolled apoptosis in primary oocytes independent of exogenous damage.

TAp63 $\beta$ forms constitutively active tetramers. TAp63a's presence from E17.5 to P10 in mouse ovaries does not impair normal follicle development in contrast to TAp63 $\beta$. In its inactive state, TAp63 $\alpha$ adopts a closed, dimeric conformation, mediated by the transactivation (TA) and transcription inhibitory (TID) $24,27,29$ domains (Fig. 4a). In case of DNA damage, TAp63a plays a protective quality control role, triggering cell death in damaged oocytes $^{8}$. Specific residues in the linker region between the SAM and TID get phosphorylated, resulting in conformational changes leading to a tetrameric active state ${ }^{29}$. As an active tetramer, TAp63a transcribes Puma and Noxa target genes to induce cell death $^{8,14}$. This regulatory mechanism is unique to TAp63a and essential for formation of inactive dimers. Consequently, in contrast to WT TAp63a, deletions in TAp63a C-terminus [(...572), (...511), (...410)] missing the TID, TAp63 $\beta$, and TAp63 $\gamma$ showed high transactivation in an in vitro transcription luciferase assay using the PUMA-responsive element in agreement with earlier reports (Fig. 4b) $)^{4,22}$. To note, the variants with higher transcriptional activity are the one detected less efficiently by western blot (Fig. 4b). As expected and shown by blue native PAGE (BNPAGE) analysis, TAp63 $\beta$ and TAp63 $\gamma$, constitute tetramers, while TAp63a is kept as a dimer (Fig. 4c, Supplementary Fig. 4a). These findings confirm that the activity of TAp63 is exclusively regulated by the oligomeric state, switching from inactive dimer to constitutively active tetramer via phosphorylation, during DNA damage in oocytes ${ }^{29}$, or lack of the TID, which is the case for all C-terminal isoforms except p63a. These data also are in agreement with the observation that only TAp63 $\alpha$ but not TAp63 $\beta$ can be detected at the protein level (Fig. 2c, g) since transcriptionally active p63 isoforms are quickly degraded while inactive ones accumulate 22,50 .

In HET $\Delta 13$ p63 mouse $\mathrm{p} 63 \alpha$ and $\mathrm{p} 63 \beta$ are expressed simultaneously, in skin as $\Delta \mathrm{Np} 63$ and in oocytes as TAp63, respectively. To investigate a potential interplay between both Cterminal isoforms, we performed in vitro transcription luciferase assays using PUMA- and NOXA-responsive elements ${ }^{51,52}$. Coexpression of TAp63a and TAp63 $\beta$ did not reduce the measured overall activity compared to only TAp63 $\beta$, suggesting that TAp63a cannot interfere with TAp63 $\beta$ transcriptional activity (Fig. 4d). This is consistent with the findings that the closed dimer of TAp63a does not interact or oligomerize with open tetramers $^{27}$. In contrast, $\Delta \mathrm{Np} 63 \alpha$ and $\Delta \mathrm{Np} 63 \beta$, expressed as tetramer (Fig. 4c), were both active when tested using envoplakin (ENV)- and bullous pemphigoid antigen 1 (BPAG1)-responsive elements $^{53}$ (Fig. 4e). Control Western blots of luciferase assay extracts are shown in Supplementary Fig. 5a, b.

Taken together, these results support the finding that while $\Delta \mathrm{Np} 63 \beta$ synergistically acts with $\Delta \mathrm{Np} 63 \alpha$ during epithelial development, TAp63 $\beta$ isoform is able to induce uncontrolled oocyte cell death independently of DNA-damage-driven phosphorylation and activation signals, causing POI in HET $\Delta 13$ p63 female mice.

Mutant TAp63a's oligomeric state determines oocyte fate. The results with the HET $\Delta 13$ p63 mouse described above have also immediate implications for the fertility of human patients suffering from different p63-related syndromes. Currently, four 
a
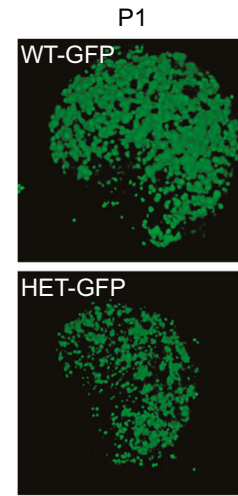

b
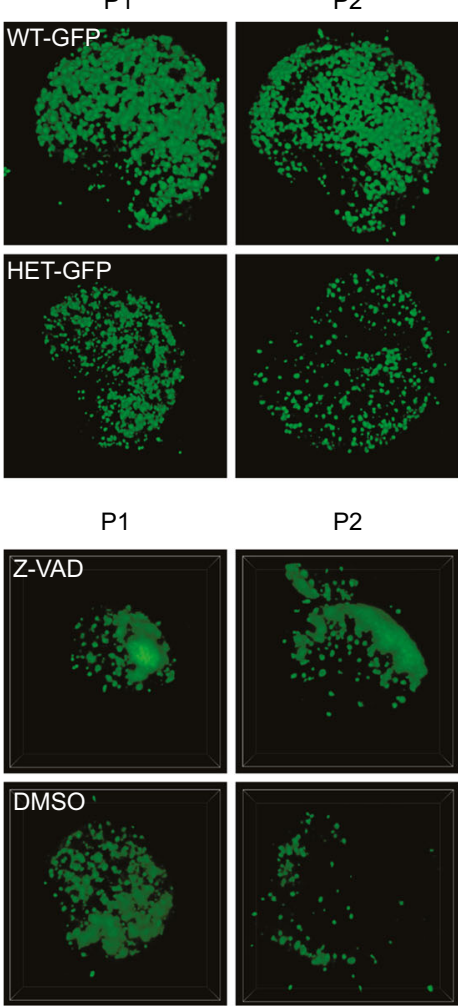

P2
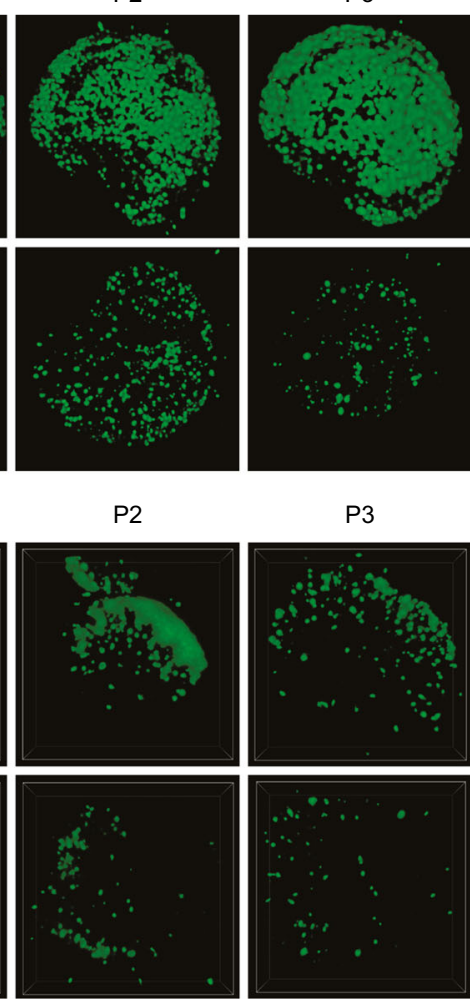

P3
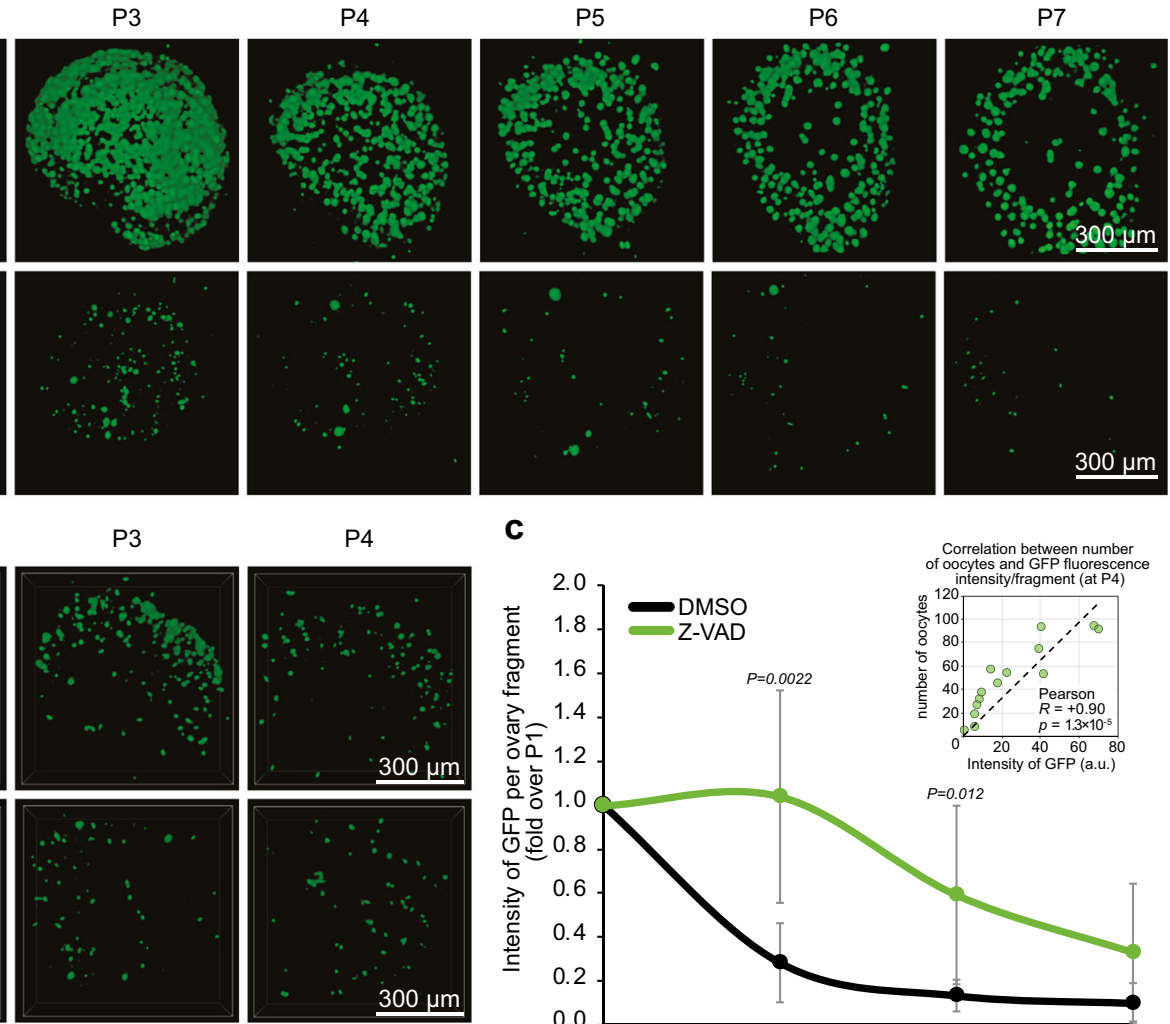

C

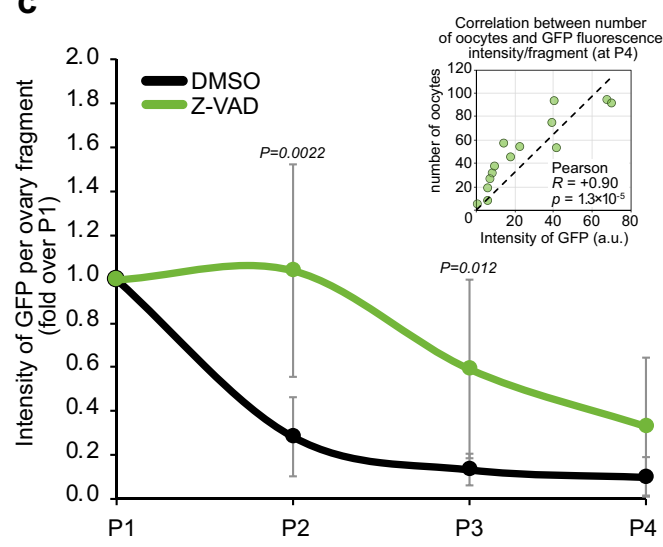

d

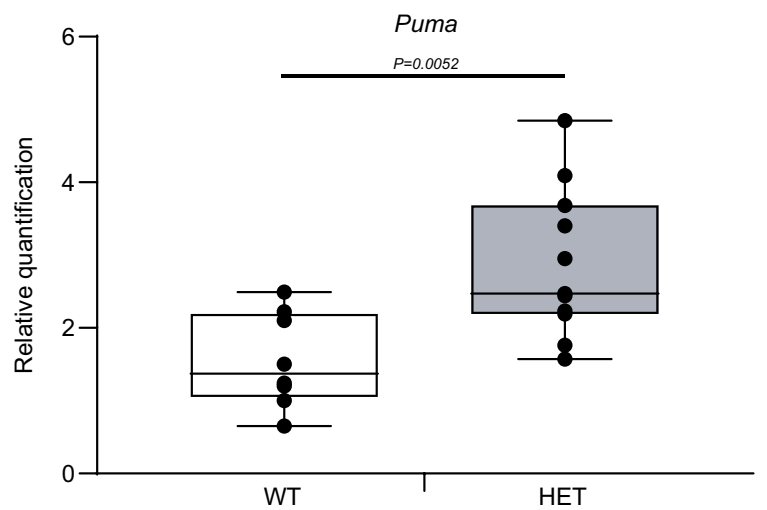

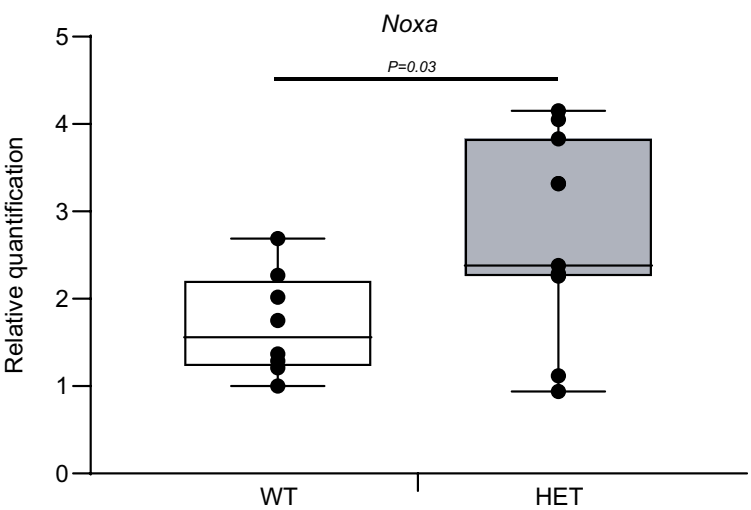

Fig. 3 The $\Delta 13 p 63$ heterozygous oocytes died by apoptosis. a Time-course (P1-P7) confocal imaging of the entire ovaries from WT-GFP and HET $\Delta 13$ p63-GFP (indicated as HET-GFP in figure) mice showing 3D rendered reconstructions using an alpha-blending algorithm. Oocytes were labelled by the expression of p-70/c-Kit fused to GFP. Images were acquired with a step distance of $5 \mu \mathrm{m}$. $\mathbf{b}$ Confocal imaging of ovarian fragments from heterozygous mice, performed as described in a. Ovaries were divided into two fragments at P1 and treated with either DMSO or $50 \mu M$ Z-VAD for three days. c Intensity of GFP/ovary fragment at P1, P2, P3, P4 in presence or absence of Z-VAD $(50 \mu \mathrm{M}) . n=7$ (DMSO) and $n=7$ (Z-VAD) ovary fragments were analysed. Data are presented as mean $\pm S D$, ${ }^{*} p$-value by two-tailed unpaired Student's $t$-test. Correlation between GFP fluorescence intensity and oocytes number (P4) is shown in the scatterplot in the upper right corner. Pearson correlation (R), between GFP fluorescence intensity/fragment and oocytes number (P4) and $p$-value are shown ( $n=7$ (DMSO) and $n=7$ (Z-VAD) ovary fragments); d Boxplots showing Puma and Noxa expression quantification by RT-qPCR in P1 ovaries of WT $(n=9)$ and HET $(n=9)$ mice. The centre of the boxplots represents median, boxes represent first $(25 \%)$ and third $(75 \%)$ quartiles, whiskers extend to the most extreme datapoints that are no more than 1.5 -fold of the interquartile range from the box. Single values are plotted as individual points. $p$-value by two-tailed unpaired Student's $t$-test. The images shown are representative of all the experiments performed (at least $n=3$ ) Source data are provided as a Source Data file.

different mutations have been reported to affect female fertility causing either POI (R555*, W559* $)^{39,40}$, premature menopause $(\text { delC1783) })^{38}$ or complete absence of ovaries (delTT1576) ${ }^{37}$. The common feature of those frameshifts and nonsense mutations is the heterozygous loss of the TID, similar to our HET $\Delta 13$ p63 mouse model. Within the ELA and AEC/RHS syndrome families many more mutations affecting the p63 C-terminus have been identified, but so far their effect on female fertility has not been investigated (Fig. 5a). To correlate the effect of the p63 mutations with the likelihood for infertility of female patients we have measured the transcriptional activity in vitro of several mutants belonging to ELA, the AEC/RHS or SHFM syndrome in reporter luciferase assays (Fig. 5b, Supplementary Fig. 4b), also including R555*, W559*, delC1783, and delTT1576.

ELA syndrome mutations can be subdivided into two different categories: point mutations within the DNA-binding domain 


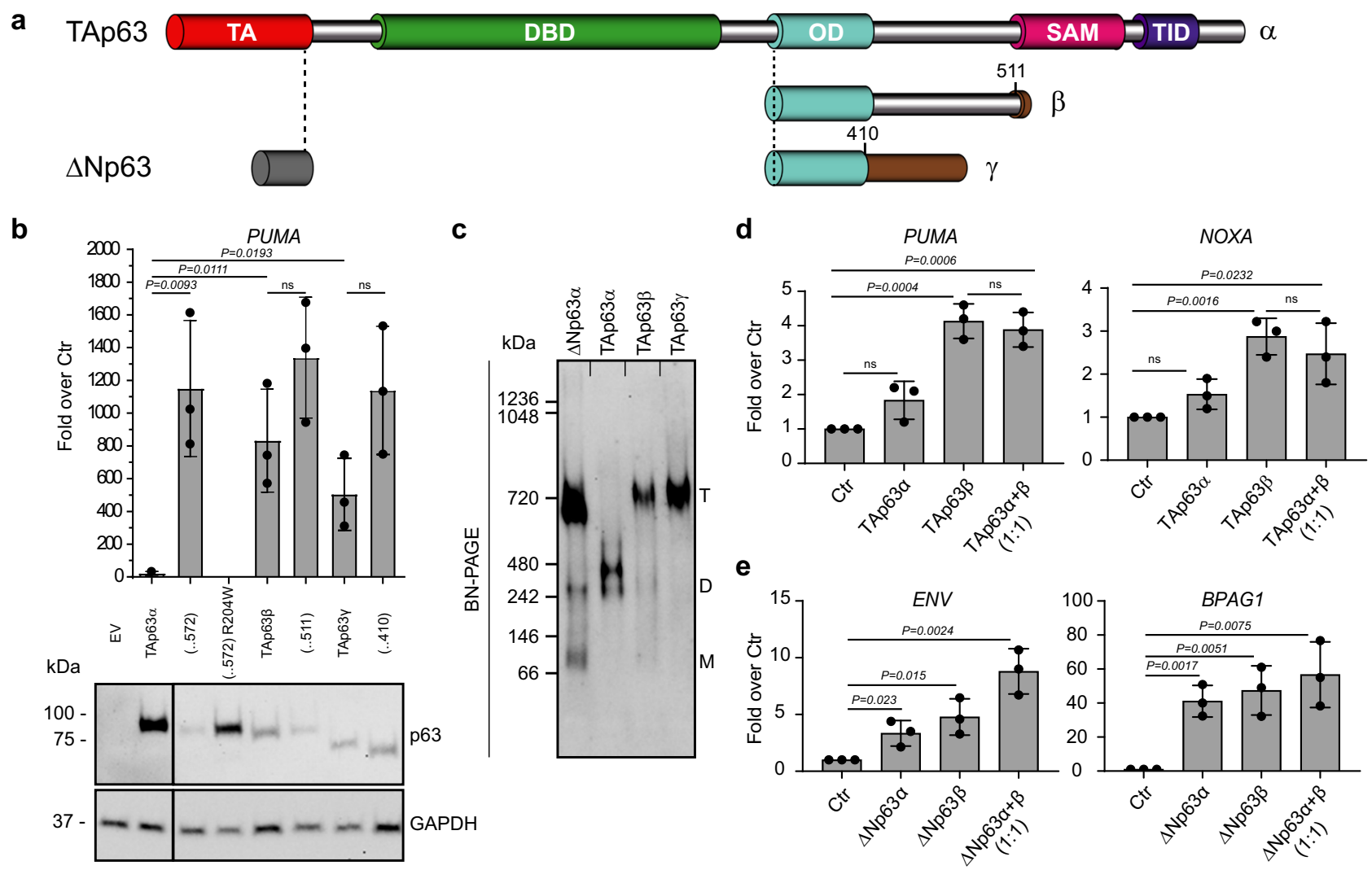

Fig. 4 The TAp63 3 isoform is a transcriptionally active tetramer. a TAp63 and $\triangle N p 63$ isoforms domains: TA transactivation domain, DBD DNA-binding domain, OD oligomerization domain, SAM sterile alpha motif, TID transactivation inhibitory domain. The C-terminus of p63 $\beta$ and p63 $\gamma$ diverge from p63 $\alpha$ starting from residue 411 or 512, respectively. b The relative luciferase activity expressed as the fold over control (Ctr) of TAp63 isoforms shows that specific C-terminus amino acids do not reduce transcriptional activity except the TID in case of the $\alpha$-isoform. TAp63 (..572) misses the post-SAM part of

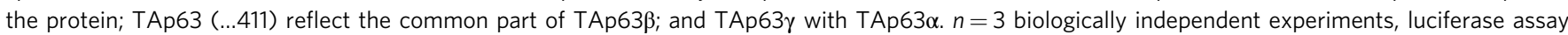
was performed in triplicates on PUMA 4xBS2WT responsive elements (REs). Data are presented as mean \pm SD, $p$-value by two-tailed unpaired Student's t-test. WB analysis of TAp63 isoforms and deletion variants in luciferase assay extracts are shown below the graph. GAPDH was used as loading control; c Oligomeric state analysis of the indicated p63 isoforms via BN-PAGE expressed in vitro in rabbit reticulocyte lysate. The oligomeric conformation is indicated by T (tetramer), D (dimer), M (monomer); d The relative luciferase activity expressed as fold over control (Ctr) shows the ability of TAp63 $\alpha$ and $\beta$ to transactivate the PUMA and NOXA promoters. $n=3$ biologically independent experiments, luciferase assay was performed in triplicates. Data are presented as mean \pm SD, $p$-value by two-tailed unpaired Student's $t$-test; $\mathbf{e}$ The relative luciferase activity expressed as the fold over control (Ctr) shows the ability of $\Delta N p 63 \alpha$ and $\Delta N p 63 \beta$ to transactivate the Envoplakin (ENV) and Bullous pemphigoid antigen 1 (BPAG1) promoters. $n=3$ biologically independent experiments, luciferase assay was performed in triplicates. Data are presented as mean \pm SD, $p$-value by two-tailed unpaired Student's $t$-test. Source data are provided as a Source Data file.

(DBD) that impair interaction with DNA (e.g. R304W) and deletions or insertions of DNA bp in the C-terminus of the protein leading to frameshifts (FSs) deleting parts of the Cterminus including the TID. As expected, R304W shows no induction in the reporter luciferase assay since this mutant is still dimeric (Fig. 5b). However, also in the tetrameric state it is not able to bind to DNA as already shown for tetrameric $\triangle \mathrm{Np} 63 \mathrm{a}^{35}$. In contrast, the ELA-FS mutations (insA1572, delTT1576, delAA1743, and delACTT1217) are transcriptionally active like TAp63 $\beta$, consistent with the absence of the inhibitory TID. Investigation of the oligomeric state further confirmed that all ELA-FS mutants are tetramers (Fig. 5c).

For the AEC mutations, the situation is more diverse. This syndrome is caused either by point mutations within the SAM (e.g. L514F) or TID or by FS mutations resulting in different Ctermini ${ }^{20}$. The common disease mechanism of all these mutations is that they expose or create aggregation prone peptide sequences that lead to aggregation and functional impairment of $\mathrm{p} 63^{36}$. The transcriptional activities of L514F within the SAM domain and FS mutations C-terminal to the TID (delA1859) are low and comparable to wild-type TAp63a (Fig. 5b), consistent with the formation of a closed dimeric state as observed by native $\mathrm{BN}$ PAGE (Fig. 5d). The remaining AEC/RHS-FS mutations ( $\triangle$ Exon11, insA1456, delG1697, delA1709, delC1721, delC1729, delC1742, delC1783, delG1787), however, are tetrameric, yet show a low transcriptional activity (Fig. 5b, d, e). All of the AEC/ RHS-FS mutations create new AA sequences with a high predicted aggregation propensity analysed by TANGO algorithm (Supplementary Fig. 6a-c). Nine of the eleven investigated AEC/ RHS-FS result in the same very C-terminal sequence (Supplementary Fig. 6c). The two AEC/RHS point mutations in the TID (R598L, D601V) show a drastic increase in aggregation propensity compared to the wild-type (Supplementary Fig. 6a).

POI mutations R555* and W559* behave similar to the tetrameric AEC/RHS-FS in the assays. They show a relatively small induction in the reporter luciferase assay compared to TAp63 3 and the ELA-FSs but are tetrameric (Supplementary Fig. $6 \mathrm{~b}, \mathrm{c})$. Due to the early stop codon in the SAM-domain sequence, this domain might not fold properly leading to exposition of aggregation prone regions (APRs) normally covered 
a

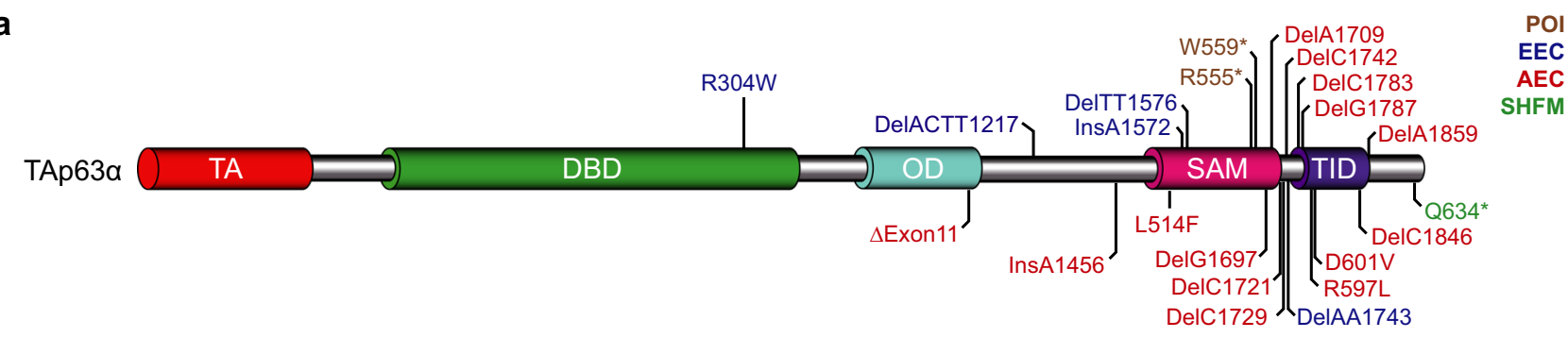

b

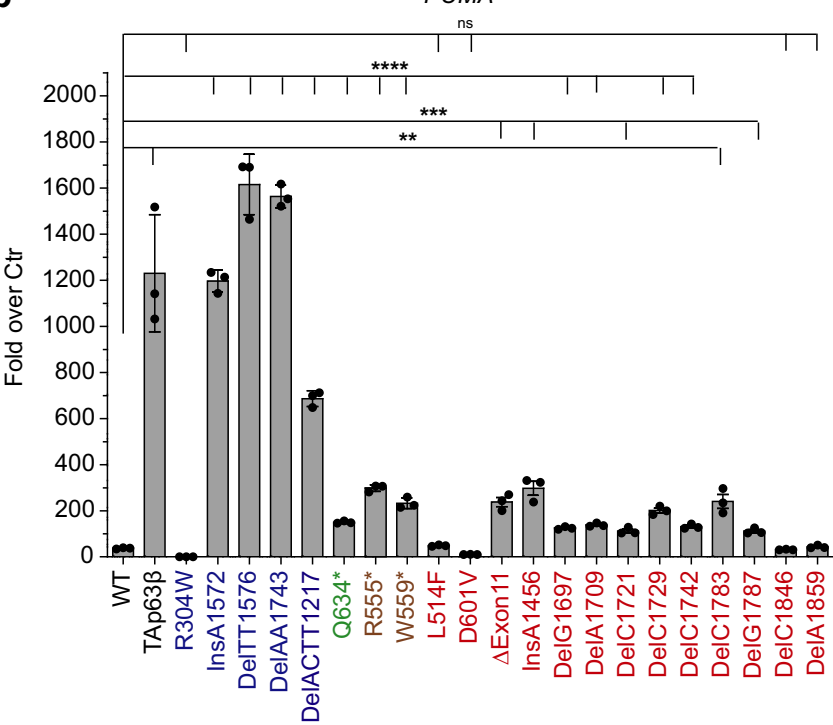

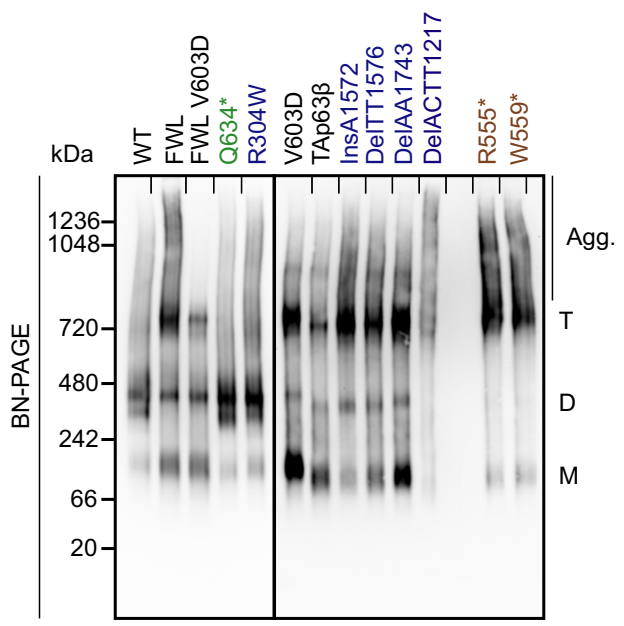

d

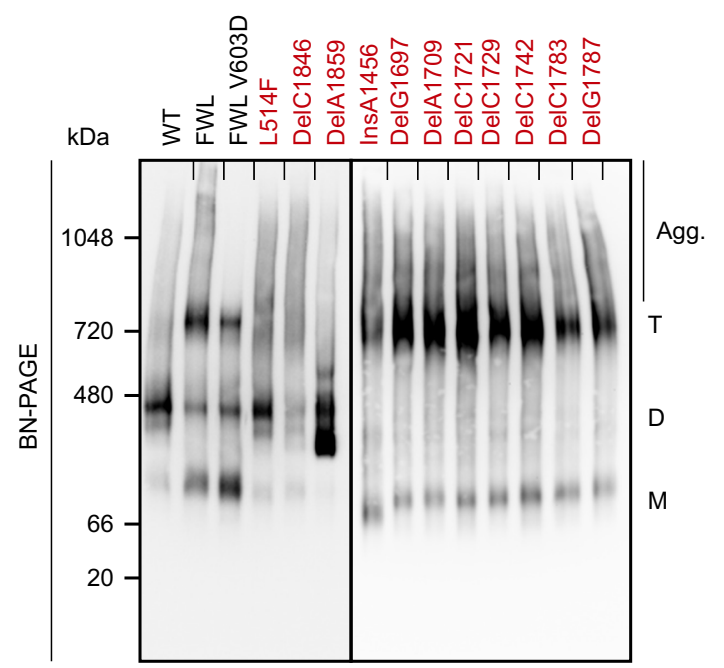

e

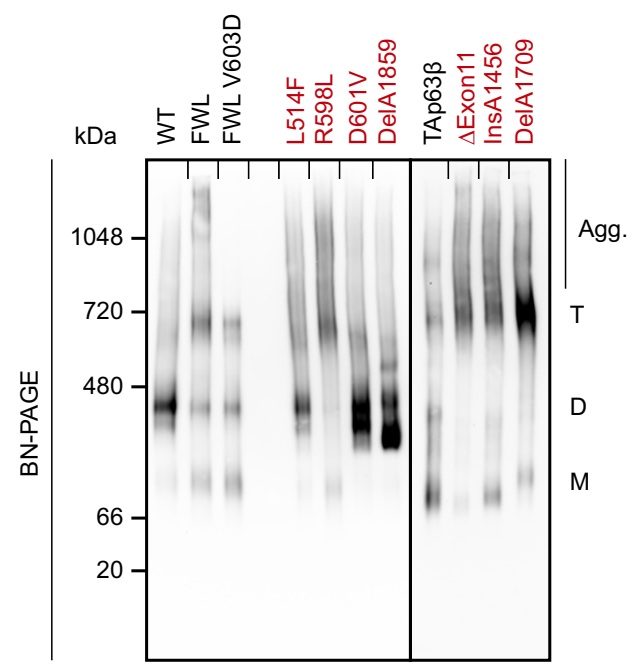

Fig. 5 Analysis of TAp63 $\alpha$ mutants' transactivation activity and oligomeric states. a Overview of different syndromic mutations on TAp63 $\alpha$. ELA mutations are represented in blue, AEC/RHS in red, SHFM in green and POI mutations in brown. $\mathbf{b}$ The relative luciferase activity expressed as fold over control (Ctr) shows the ability of wild-type or mutant p63 variants to transactivate the PUMA promoters. $n=3$ biologically independent experiments, luciferase assay was performed in triplicates on PUMA 4xBS2WT responsive elements (REs). Data are presented as mean \pm SD, ${ }^{\star \star} p$-value $<0.01$, ${ }^{\star * \star} p$-value $<0.001,{ }^{* \star *} p$-value $<0.0001$, ns $p$-value $>0.05$ by two-tailed unpaired Student's $t$-test. Exact $p$-values are provided in Source Data file; c-e Oligomeric state analysis of mutant TAp63 $\alpha$ via blue native (BN)-PAGE. The oligomeric conformation is indicated by T (tetramer), D (dimer), $M$ (monomer) and, additionally, Agg. for aggregation. Source data are provided as a Source Data file.

in the hydrophobic core of the SAM. To confirm this suggestion, we mutated the aggregation prone regions by either introducing aspartic acid residues (mutAPR) or by simply deleting the region $(\triangle \mathrm{APR})$ in the two p63a POI and selected AEC/RHS mutants, analyzed by TANGO algorithm (Supplementary Fig. 6d-g). The results of the transactivation assays show that all tetrameric AEC/ RHS or POI mutants reach high transcriptional activity when aggregation is suppressed (Supplementary Fig. 7a, b) like TAp63 $\beta$ and the ELA-FS mutations. On BN-PAGE, strongly reduced aggregation is visible in case of the rescue deletions (Supplementary Fig. 7c, d).

These results show that FS mutations disrupting the inactive dimeric state of TAp63a are likely to cause POI condition in female patients suffering from developmental defects in epithelial tissues and appendages caused by the same mutations in the $\triangle \mathrm{Np} 63 \mathrm{a}$ isoform. However, there is a significant difference 
Table 1 Summary table of predicted oocyte fate.

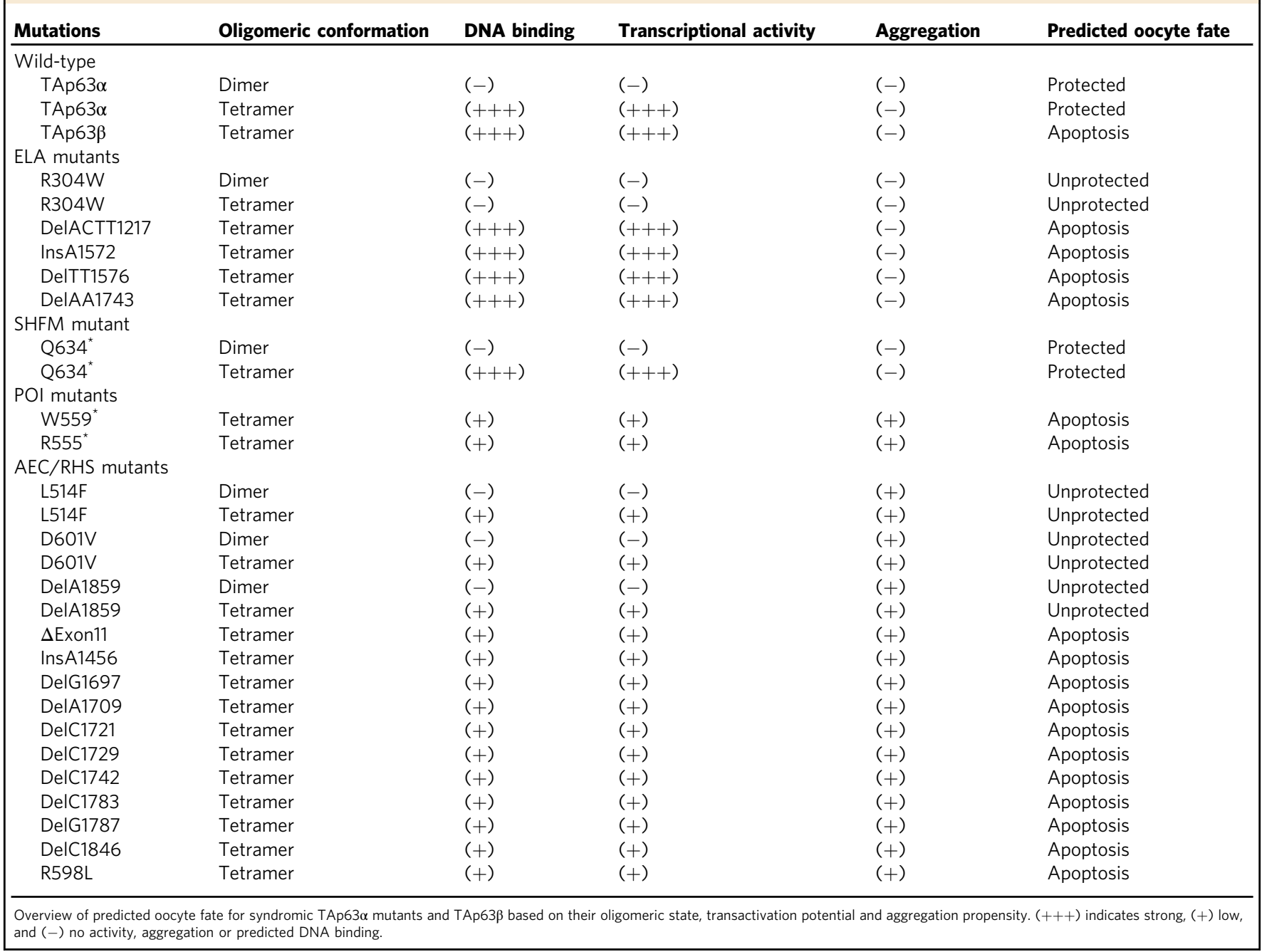

between tetrameric ELA- and AEC/RHS-p63a mutations. The low activity of AEC/RHS mutations due to aggregation reflects the temporally delayed effect of premature menopause while the tetrameric ELA-FS, like TAp63 $\beta$ in the HET $\Delta 13 p 63$ mouse, would probably kill all oocytes shortly after their expression. The effect of mutations not disturbing the inactive dimer (R304W, L514F, delA1859, and Q634*) will most likely only emerges once TAp63 $\alpha$ gets activated by DNA damage. Inhibition of DNA binding or aggregation could then delay or impair apoptosis, cancelling TAp63a's role as the guardian of the oocyte genome. An overview of these results and the predictions for the individual syndromes is summarized in Table 1 .

\section{Discussion}

The roles of TAp63 and $\Delta \mathrm{Np} 63$ in vivo have been previously investigated by generating selective knockout mice for either isoform, resulting in the identification of distinct phenotypes $^{11,12,54}$. However, aside from early controversial work on exon $12^{25}$, the specific role of the p63 C-terminus in vivo remains largely unexplored. The p63a isoform is by far the most abundant p63 isoform in epithelial tissues $(\triangle \mathrm{Np} 63)$ and in primary oocytes (TAp63). Here, using a genetic mouse model, we knocked out the p63a isoform, replacing it with p63 $\beta$. The results showed a profound phenotype in heterozygous females leading to a condition of ovary disfunction resembling POI in human females, consistent with descriptions of p63 mutations in human patients affecting fertility (Fig. 6) $37,38,40$.

Recently, a whole-genome sequencing investigation of 13 POI patients demonstrated that TAp63 truncated variants in the terminal exon 14 are associated with female infertility ${ }^{40}$. Both variants identified in this study, located in the SAM (R555* and W559*), generate a p63-truncated isoform lacking the TID and the C-terminal part of the SAM. This truncation is responsible for the inability of the SAM domain to adopt its native structure exposing two aggregation prone regions usually hidden in the fold. The POI mutants are tetrameric but show a limited activity due to APR exposure compared to TAp63 $\beta$. The reduced activity results in a slow depletion of the oocyte pool over time. The absence of a typical AEC syndrome phenotype of the patients, however, cannot be explained.

In contrast to the POI mutants, the AEC/RHS-FS mutations create new aggregation peptides not present in the wild-type protein. The FS mutation delC1783, described to induce premature menopause around the age of 30 years ${ }^{38}$, shares the same C-terminal frame with eight of the eleven investigated AEC/RHSFS mutants. For all tetrameric FS mutants, we would expect a similar oocyte fate of slow oocyte elimination by apoptosis. Further effects on female fertility are not described for AEC/RHS patients. On the contrary, the tetrameric ELA-FS mutants are likely to result in complete destruction of all oocytes before puberty. The results of the reporter luciferase assay suggest that 

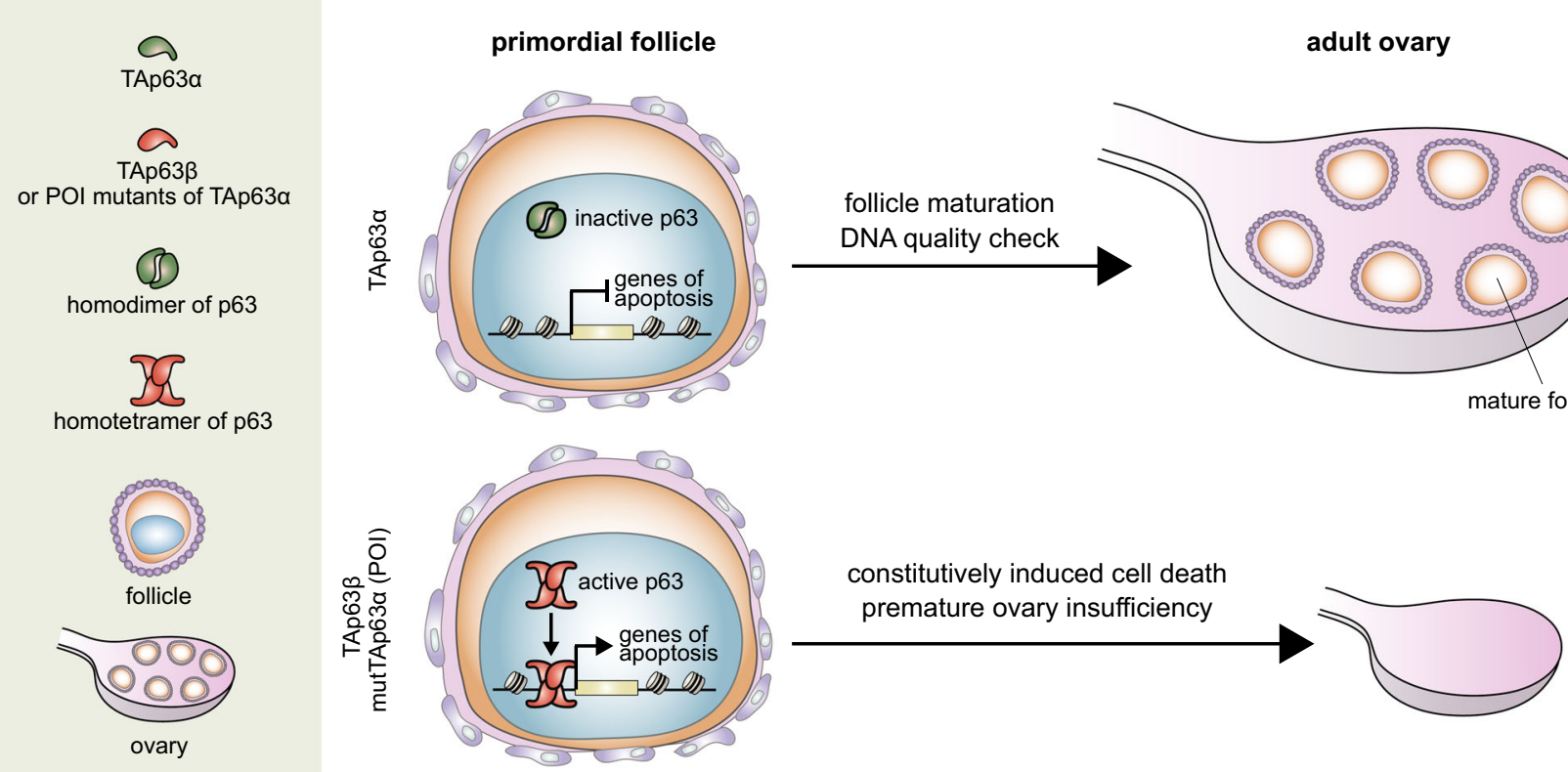

Fig. 6 Schematic model of TAp63 role in ovary physiology, in HET $\Delta 13 p 63$ mouse model and in presence of POI mutations. Under normal physiological conditions, TAp63 $\alpha$ is present in dimeric inactive form, which can be activated only upon certain circumstances such us upon DNA damage. Activated TAp63 $\alpha$ enables DNA quality check to ensure the genomic stability of germline ovary development and follicle maturation. However, TAp63 $\beta$, as well as $\mathrm{POI}$ mutants of $\mathrm{p} 63$, form active tetramers which constitutively induce cell death, leading to premature ovarian insufficiency.

they induce uncontrolled expression of proapoptotic genes, initiating apoptosis of the oocyte similar to TAp63ß.

In case of syndromic, still dimeric TAp63a mutants (R304W, delA1859, D601V, and Q634*), the survival of the oocytes should not be influenced. However, in presence of DNA damage, the quality control mechanism of TAp63a can be delayed or completely disturbed. After phosphorylation, TAp63a mutants cannot initiate directly transcription of proapoptotic genes because they are either incapable to bind to DNA or their activity is suppressed by protein aggregation.

Several studies have reported the detection of p63 (and in particular TAp63a) in male germ cells. Mice with a Trip13 mutation, a conserved AAA + ATPase required for the completion of meiotic DSB repair, arrest spermatocytes at the pachytene stage of meiosis caused by unrepaired DNA double-strand breaks. Inactivation of either p53 or TAp63 in these mice, however, enables spermatocytes to further progress while inactivation of the third family member, p73, has no effect. It was further shown that this pachytene cell-cycle arrest in spermatocytes of Trip13 mutant mice can be prevented by eliminating the kinase ATM or the ATM-effector kinase Chk2 which in the female germline are essential for the activation of TAp63a ${ }^{55,56}$. Despite these previous findings, our HET $\triangle 13$ p63 mouse does not show an effect on male fertility. If TAp63a in male germ cells constitutes a similar quality control check point as in female germ cells the expression of the constitutively active TAp63 should eliminate all spermatocytes passing through the pachytene check point. The uncompromised fertility of the male HET $\Delta 13 p 63$ mice raises questions about the role of TAp63a during spermatogenesis and calls for a more detailed analysis including the quantity and quality of sperm cells produced in these mice.

In humans and great apes yet another isoform of p63 has been identified, called GTAp63a. This isoform is created by the by insertion of the LTR region of the human endogenous retrovirus 9 upstream of the TAp63 gene resulting in the addition of 37 amino acids at the $\mathrm{N}$-terminus. A detailed biochemical analysis of this isoform has shown that this additional sequence results in a further stabilization of the dimeric, inactive state ${ }^{16}$. Why (human) male germ cells require this extra safety mechanism remains an open question but it has been proposed that expression of GTAp63a is important to enable longer reproductive periods in hominids. The different roles that p63 isoforms play in female and male germ cell quality control also reflect the different strategies used to produce these cells. In the female germline stem cells are quiescent and a limited number of arrested germ cells constitutes the ovarian reserve. In contrast, in the male germline stem cells are actively dividing resulting in the mass production of sperm cells. Differences in quality surveillance are also manifested in the observation that disease causing point mutations disproportionately arise from the male germline, whereas chromosomal aberrations are mostly transmitted by female germ cells.

While TAp63 $\beta$ causes female infertility in HET $\Delta 13 p 63$, replacement of $\Delta \mathrm{Np} 63 \alpha$ with $\Delta \mathrm{Np} 63 \beta$ in other tissues/organs does not cause morphological or functional defects. This is probably because $\Delta \mathrm{Np} 63 \alpha$ and $\Delta \mathrm{Np} 63 \beta$ are tetramers and act synergistically to control target gene expression, acting both as repressors and activators. However, we must keep in mind that potential as-yet unidentified SAM-domain interactors or posttranslational modifications, such as sumoylation/ubiquitylation occurring at the p63 C-terminal lysine residues $23,26,57,58$, are abrogated in the $\Delta \mathrm{Np} 63 \beta$ isoform, leaving open the possibility that under particular stress/pathological conditions, epithelial tissues lacking $\triangle \mathrm{Np} 63 \alpha$ could be defective.

Although TAp63 and $\Delta \mathrm{Np} 63$ isoforms play roles in mouse embryo development and adult lifespan regulation, the relative contributions and roles of their C-termini have not been conclusively established in a defined in vivo genetic system. The generation and characterization of HET $\Delta 13 \mathrm{p} 63$ mice clearly contributed to understand the molecular basis and the biological importance of TAp63 regulation for oocytes quality control. Further studies, based on exon 13 conditional deletions, will help in determing the role of $\mathrm{p} 63 \mathrm{C}$-terminus in other p63-expressing tissues. Our results show that the TID inhibitory effect is indispensable in TAp63a-expressing primary oocytes to control cell death in vivo, supporting and expanding the current knowledge regarding human primary ovarian insufficiency. 


\section{Methods}

Generation of $\Delta 13 p 63$ heterozygous mice and maintenance of the mouse

colony. P63 exon 13 floxed allele mice were generated by Ozgene (Ozgene Pty Ltd, Bentley, Australia), and the floxed/ + colony was amplified by crossing with wildtype $\mathrm{C} 57 \mathrm{Bl} / 6 \mathrm{~J}$ mice. Heterozygous floxed/+ mice were crossed with $\mathrm{Tg}(\mathrm{CMV}$-cre) 1 Cgn homozygous mice ${ }^{59}$ to obtain the p63 exon 13-deleted allele ( $\left.\Delta 13 \mathrm{p} 63\right)$. The $\Delta 13 \mathrm{p} 63 / \mathrm{CMV}$-Cre heterozygous mice were backcrossed with wild-type C57Bl/6 mice to lose the CMV-Cre allele. The pure $\Delta 13$ p63 heterozygous mice obtained were crossed as described in Table S1. Transgenic $\mathrm{p}-70 \mathrm{GFP} / \mathrm{c}-\mathrm{Kit}^{60}$ female mice were crossed with $\Delta 13 \mathrm{p} 63$ heterozygous male mice to obtain $\mathrm{p}-70 \mathrm{GFP} / \mathrm{c}-\mathrm{Kit}$ $\Delta 13$ p63 heterozygous ovaries. The mouse genotypes were assessed by PCR using the primers listed in Supplementary Table 1. The mice were bred in-house and housed in a temperature- and light-controlled mouse colony room (12-h light/dark cycle) and had free access to food and water. All experiments were approved by the Institutional Animal Care and Use Committee (IACUC) and were carried out according to the Italian and European rules (D.L.116/92; C.E. 609/86; European Directive 2010/63/EU). For mice experiments licence $n^{\circ}$ 817/2016PR (Italian Ministry of Health).

Cell cultures, transfections, and luciferase assays. HEK293 and H1299 cell lines were grown in Dulbecco's modified Eagle's medium (Thermo Fisher, Waltham, MA, USA) supplemented with $10 \%$ (vol/vol) foetal bovine serum (Thermo Fisher, Waltham, MA, USA) and $1 \%$ penicillin/streptomycin (Thermo Fisher, Waltham, MA, USA) at $37^{\circ} \mathrm{C}$ in a humidified atmosphere of $5 \%$ (vol/vol) $\mathrm{CO}_{2}$ in air.

A total of $1.2 \times 10^{5}$ HEK293 cells were seeded in 12 -well dishes $24 \mathrm{~h}$ before transfection. A total of $100 \mathrm{ng}$ of pGL3 vectors, $300 \mathrm{ng}$ of transactivators expression vectors and $10 \mathrm{ng}$ of Renilla luciferase pRL-CMV vector (Promega, Madison, WI, USA) were cotransfected using Effectene Reagent (Qiagen, Hilden, Germany) according to the manufacturer's protocol. pGl3 vectors containing p63-responsive elements in ENV and BPAG1 promoters ${ }^{61}$.

Puma and Noxa p63-responsive elements described before ${ }^{14}$ were amplified by PCR from human genomic DNA and cloned in pGL3basic vector (Promega, Madison, WI, USA). Relative luciferase activities were measured $24 \mathrm{~h}$ after transfection using a Dual Luciferase Reporter Assay System (Promega, Madison, WI, USA). Light emission was measured over $10 \mathrm{~s}$ using a Lumat LB9507 luminometer (EG\&G Berthold, Wildbad, Germany). The efficiency of transfection was normalized to Renilla luciferase activity.

A total of $0.8 \times 10^{5} \mathrm{H} 1299$ cells were seeded in a 12 -well plate $24 \mathrm{~h}$ before transfection. $267 \mathrm{ng}$ of vectors Puma $4 \times x_{\text {BS2WT-Luc }}{ }^{62}$ (kind gift from B.

Vogelstein), pcDNA3, and pRL-CMV were cotransfected using the Lipofectamine ${ }^{\oplus}$ 2000 according to manufacturer's manual and grown for $24 \mathrm{~h}$. Relative luciferase activities were measured as described before. Measurement was performed at Tecan Spark multimode microplate reader (Tecan, Männedorf, Switzerland). A total of $4 \times 10^{5} \mathrm{H} 1299$ cells were seeded $24 \mathrm{~h}$ before transfection. The same amount of HA tagged TAp63 $\alpha$ and TAp63 $\beta$ expression vectors, or empty vector as control, were cotransfected using Effectene Reagent (Qiagen, Hilden, Germany) according to the manufacturer's protocol. $24 \mathrm{~h}$ after transfection, medium was removed and replaced with complete fresh medium. $10 \mu \mathrm{M}$ MG132 or DMSO as control were added. After $1 \mathrm{~h}$, cycloheximide treatment $(50 \mu \mathrm{g} / \mathrm{ml})$ was started and samples were collected at $0,2,4,6$, and $8 \mathrm{~h}$.

Primary mouse keratinocytes were isolated from newborn mouse skin and collected in proliferation conditions.. Briefly newborn mouse pups were sacrified, epidermises were separated from dermis by overnight incubation in trypsin, then they were minced with scissors, triturated by pipetting several times. Single cells were filtred through a $100-\mu \mathrm{m}$ cell strainer (Corning, Glendale, AZ, USA),

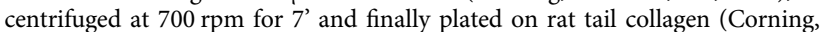
Glendale, AZ, USA) coated dishes in low calcium medium: EMEM 06-174G (LONZA Bioscience, Basel, Switzerland), 8\% chelate FBS, antibiotic/antimycotic solution (Sigma, St. Louis, MO, USA) and $0,05 \mathrm{mM} \mathrm{CaCl}_{2}$. Twenty-four hours later, aderent cells were washed three times in PBS and then collected to be processed as desired.

RNA extraction and real-time PCR analysis. Total RNA was isolated from mouse tissues by RNeasy Mini Kit (Qiagen, Hilden, Germany) and retrotranscribed by GoScript Reverse Transcription System (Promega, Madison, WI, USA) according to manufacturer's protocol. Real Time PCR was performed using GoTaq qPCR Master Mix (Promega, Madison, WI, USA). Primers used are listed in Supplementary Table 1 . The expression of each gene was defined from the threshold cycle $(\mathrm{Ct})$ and the relative expression levels were calculated by using the $2^{-\Delta \Delta \mathrm{Ct}}$ method after normalization with reference to expression of $\beta$-actin as housekeeping gene. Endpoint semi-quantitative PCR were performed using GoTaq polymerase (Promega, Madison, WI, USA) according to manufacturer protocol. Primers used are listed in Supplementary Table 1.

Histology and immunofluorescence. Freshly isolated mouse skin and thymus samples were embedded in OCT (Bio-Optica, Milano, Italy), cryosectioned $(10 \mu \mathrm{m})$ and section fixed in $4 \%$ paraformaldehyde for 10 ' at room temperature. Testes and ovaries were fixed directly in $4 \%$ paraformaldehyde $(12-16 \mathrm{~h})$, then embedded in paraffin and H/E or PAS stained following standard procedures.
For immunofluorescence (IF) staining, cryosections $(10 \mu \mathrm{m})$ were fixed in $4 \%$ paraformaldehyde for $10^{\prime}$ at room temperature. Nonspecific antigens were blocked by incubation in 5\% goat serum in PBS for $1 \mathrm{~h}$ in a humidified atmosphere at room temperature. Subsequently, the sections were incubated with primary antibodies for $2 \mathrm{~h}$ at room temperature. The sections were then washed three times with PBS and incubated for $1 \mathrm{~h}$ with the appropriate secondary antibodies conjugated with Alexa Fluor 488 or 568 (Thermo Fisher, Waltham, MA, USA) and DAPI. The slides were then mounted using Prolong Antifade (Thermo Fisher, Waltham, MA, USA) IF images were acquired with a Nikon A1 confocal laser microscope (Nikon, Tokyo, Japan).

For follicle counting, whole P1 ovaries were sectioned and stained with an antiMSY2 primary antibody. All MSY2-positive oocytes were counted in every fifth section throughout the entire ovary, and the mean total number of oocytes/sections was multiplied by the total number of sections of each ovary.

The catalogue numbers and sources of primary antibodies were as follows: antiK5 (PRB-160P BioLegend; San Diego, CA, USA; 1/1000), Troma-1 (anti-K8, Developmental Studies Hybridoma Bank, Iowa City, IA, USA; 1/2000), anti-K14 (PRB-155P BioLegend; San Diego, CA, USA; 1/1000), anti-K10 (PRB-159P BioLegend; San Diego, CA, USA; 1/1000), anti Ki67 (91295-D3B5 Cell Signaling Technologies, Danvers, MA, USA; $1 / 200$ ), anti $\beta 4$ integrin (611232, BD Biosciences, San Jose, CA, USA), anti-p63 (D9L7L, Cell Signaling Technologies, Danvers, MA, USA; 1/200), and Msy2 (A12 Santa Cruz Biotechnologies, Dallas, TX, USA; 1:2000).

Ovary culture. Ovaries were collected at P1 from p-70 GFP/c-Kit mice and cultured in tissue culture four-well plates in $0.3 \mathrm{ml}$ of a-MEM supplemented with $10 \%$ FBS, L-glutamine, penicillin-G, streptomycin, pyruvic acid, $\mathrm{N}$-acetyl-L-cysteine and ITS liquid medium supplement at $37^{\circ} \mathrm{C}$ in $5 \% \mathrm{CO}_{2}$ for a maximum of 7 days; the medium was changed every 2 days. In the experiments in which the dynamics of oocytes degeneration was followed in the presence of $50 \mu \mathrm{M}$ Z-VAD-FMK or DSMO, in order to favour the inhibitor diffusion, each ovary was sliced into four pieces and cultured for 4 days under the conditions reported above except that medium was changed every day. Ovaries and fragments were then analysed for 3D rendered confocal imaging. Z-stacks were acquired every $5 \mu \mathrm{m}$ for a total of $300 \mu \mathrm{m}$, and 3D rendering was performed with NIS Elements software (Nikon, Tokyo, Japan) using the alpha-blending algorithm. To estimate the number of oocytes in the experiment with Z-VAD treatment, the sum intensity of GFP fluorescence per fragment was quantified for each timepoint (from P1 to P4) using NIS Elements software. Intensity values per fragment for each timepoint were normalised to the intensity values at P1. Pearson coefficient (R) was calculated to confirm the correlation between intensity of GFP fluorescence and oocytes number. Manually counted oocytes at P4 were used for correlation analysis.

Western blotting. Cell and tissues extracts were resolved on SDS polyacrylamide gels and blotted onto Hybond P, PVDF membrane (GE Healtcare Chicago, IL, USA). Membranes were blocked with PBST 5\% non-fat dry milk, incubated with primary antibodies for $2 \mathrm{~h}$ at room temperature, washed and hybridized with peroxidase conjugated secondary antibodies for $1 \mathrm{~h}$ at room temperature (goat anti-rabbit or goat anti-mouse, Biorad Hercules, CA, USA). Detection was performed with the ECL chemiluminescence kit (Perkin Elmer, Waltham, MA, USA) The antibodies used were: anti-p63a (D2K8X, Cell Signaling Technologies, Danvers, MA, USA; 1/500 dilution), anti-p63 (D9L7L, Cell Signaling Technologies, Danvers, MA, USA; 1/250), anti-GAPDH (clone 6C5, Merck Millipore, Darmstadt Germany), anti-HA.11 (Biolegend, San Diego, CA, USA; 1/500), and anti- $\beta$ actin (AC-15, Sigma, St. Louis, MO, USA; 1/5000)

Uncropped blots for westerns and other blots can be found in the Source data file.

In vitro and mammalian cell expression followed by blue native PAGE (BNPAGE). For BN-PAGE, the different p63 isoforms/mutants were expressed either in H1299 cells or using the TNT ${ }^{\oplus}$ T7 Coupled Reticulocyte Lysate System (Promega, Madison, WI, USA) following manufacturer's protocol. For expression in H1299 cells, cells were transfected, harvested and lysed in $1 \times$ BN-PAGE lysis buffer (50 mM Tris pH 8.0, $100 \mathrm{mM} \mathrm{NaCl}, 20 \mathrm{mM}$ CHAPS, $0.5 \mathrm{mM}$ TCEP, $2 \mathrm{mM} \mathrm{MgCl}_{2}$ $1 \times$ cOmplete (Roche, Basel, Switzerland), and $1 \times$ PhosSTOP (Roche, Basel, Switzerland) with $1 \mu \mathrm{l}$ Benzonase (Merck) added on ice for $1 \mathrm{~h}$. The supernatant was supplemented with $3 \times$ Native PAGE sample buffer $(60 \%$ glycerol v/v, $15 \mathrm{mM}$ coomassie G250) and samples were analysed using the BN-PAGE Novex 3-12\% Bis-Tris protein gel system (Life Technologies) according to the manufacturer's instructions. The cathode buffer was supplemented with $0.002 \%$ coomassie G250, and the separation was performed at $4{ }^{\circ} \mathrm{C}$ for $60 \mathrm{~min}$ at $150 \mathrm{~V}$ followed by $60 \mathrm{~min}$ at $250 \mathrm{~V}$. The BN-PAGE was transferred onto a $0.45 \mu \mathrm{m}$ PVDF membrane using a tank-blot method from Life Technologies (XCell II blot module) according to the manufacturer's instructions. Proteins were transferred at $30 \mathrm{~V}$ for $105 \mathrm{~min}$ at $4^{\circ} \mathrm{C}$ with precooled $1 \times \mathrm{NuPAGE}^{\mathrm{TM}}$ Transfer buffers. After the transfer, the membrane was incubated for $15 \mathrm{~min}$ in $8 \%$ acetic acid and afterwards destained using $100 \%$ methanol. For in vitro expression, $20 \mu \mathrm{l}$ of reticulocyte lysate was mixed with $1 \mu \mathrm{g}$ of DNA in a total volume of $25 \mu \mathrm{l}$. After $90^{\prime}$ at $30^{\circ} \mathrm{C}, 20 \mu \mathrm{l}$ of the reaction was mixed with $100 \mu \mathrm{l}$ of $2 \times$ native PAGE buffer in a total volume of $200 \mu \mathrm{l}$. Then, $10 \mu \mathrm{l}$ 
of this mixture was mixed with $5 \mu$ l of $3 \times$ native PAGE loading buffer ( $60 \%$ glycerol and $15 \mathrm{mM}$ Coomassie G250). A total of $7.5 \mu$ of each final sample was subsequently used for native PAGE and western blot analysis, as previously described ${ }^{29}$. The p63 isoforms /mutants were detected using an N-terminal Myc-Tag (anti-Myc antibody 4A6, Millipore, Burlington, MA, USA) or a p63-specific antibody (ab124762, Abcam, Cambridge, UK).

In silico analysis of aggregation propensity. TANGO algorithm (http://tango. crg.es/) was used to investigate the $\beta$-aggregation propensity of wild-type and developmental syndromic mutants of p63a. The following parameters were chosen for analyzation: $\mathrm{pH} 7.2$, temperature of $4^{\circ} \mathrm{C}$ and ionic strength $0.15 \mathrm{M}$.

Statistics and reproducibility. Data are shown as individual values and/or means \pm Standard deviation (SD), if not otherwise stated. Experimental results significance was evaluated by the Student's $t$-test, differences with $p<0.05$ were considered significant. Statistics in unpaired Student's $t$-test, Chi-square test and Pearson correlation analysis were performed by GraphPad Prism 7.0 software (GraphPad Software Inc., San Diego, CA, USA). All experiments were performed at least three times.

Reporting summary. Further information on research design is available in the Nature Research Reporting Summary linked to this article.

\section{Data availability}

All data generated or analysed during this study are included in this published article and its Supplementary Information files. Source data are provided with this paper.

Received: 6 February 2020; Accepted: 26 November 2020; Published online: 15 January 2021

\section{References}

1. Mills, A. A. et al. P63 is a P53 homologue required for limb and epidermal morphogenesis. Nature 398, 708-713 (1999).

2. Yang, A. et al. p63 is essential for regenerative proliferation in limb, craniofacial and epithelial development. Nature 398, 714-718 (1999).

3. Yang, A. \& McKeon, F. p63 and p73: p53 mimics, menaces and more. Nat. Rev. Mol. Cell Biol. 1, 199-207 (2000).

4. Yang, A. et al. p63, a p53 homolog at 3q27-29, encodes multiple products with transactivating, death-inducing, and dominant-negative activities. Mol. Cell $\mathbf{2}$, 305-316 (1998).

5. Duijf, P. H. G. Gain-of-function mutation in ADULT syndrome reveals the presence of a second transactivation domain in p63. Hum. Mol. Genet. 11, 799-804 (2002).

6. Rufini, A. et al. Differential roles of p63 isoforms in epidermal development: selective genetic complementation in p63 null mice. Cell Death Differ. 13, 1037-1047 (2006).

7. Crum, C. P. \& McKeon, F. D. p63 in epithelial survival, germ cell surveillance, and neoplasia. Annu. Rev. Pathol. Mech. Dis. 5, 349-371 (2010).

8. Suh, E. K. et al. p63 protects the female germ line during meiotic arrest. Nature 444, 624-628 (2006)

9. Levine, A. J., Tomasini, R., McKeon, F. D., Mak, T. W. \& Melino, G. The p53 family: guardians of maternal reproduction. Nat. Rev. Mol. Cell Biol. 12, 259-265 (2011).

10. Su, X. et al. TAp63 is a master transcriptional regulator of lipid and glucose metabolism. Cell Metab. 16, 511-525 (2012).

11. Romano, R.-A. et al. $\Delta \mathrm{Np} 63$ knockout mice reveal its indispensable role as a master regulator of epithelial development and differentiation. J. Cell Sci. 139, 772-782 (2012).

12. Su, X. et al. TAp63 Prevents premature aging by promoting adult stem cell maintenance. Cell Stem Cell 5, 64-75 (2009).

13. Gonfloni, S. et al. Inhibition of the c-Abl-TAp63 pathway protects mouse oocytes from chemotherapy-induced death. Nat. Med. 15, 1179-1185 (2009).

14. Kerr, J. B. et al. DNA damage-induced primordial follicle oocyte apoptosis and loss of fertility require TAp63-mediated induction of Puma and Noxa. Mol. Cell 48, 343-352 (2012).

15. Beyer, U., Moll-Rocek, J., Moll, U. M. \& Dobbelstein, M. Endogenous retrovirus drives hitherto unknown proapoptotic p63 isoforms in the male germ line of humans and great apes. Proc. Natl Acad. Sci. USA 108, 3624-3629 (2011).

16. Pitzius, S. et al. TA* ${ }^{*} 63$ and GTAp63 achieve tighter transcriptional regulation in quality control by converting an inhibitory element into an additional transactivation domain. Cell Death Dis. 10, 686 (2019).

17. Petre-Lazar, B. et al. The role of $\mathrm{p} 63$ in germ cell apoptosis in the developing testis. J. Cell. Physiol. 210, 87-98 (2007).
18. Bornstein, C. et al. SPATA18, a spermatogenesis-associated gene, is a novel transcriptional target of p53 and p63. Mol. Cell. Biol. 31, 1679-1689 (2011).

19. Wang, H. et al. Transcriptional regulation of P63 on the apoptosis of male germ cells and three stages of spermatogenesis in mice. Cell Death Dis. 9, 76 (2018).

20. McGrath, J. A. Hay-Wells syndrome is caused by heterozygous missense mutations in the SAM domain of p63. Hum. Mol. Genet. 10, 221-229 (2001).

21. Ou, H. Der, Löhr, F., Vogel, V., Mäntele, W. \& Dötsch, V. Structural evolution of C-terminal domains in the p53 family. EMBO J. 26, 3463-3473 (2007).

22. Serber, $Z$. et al. A C-terminal inhibitory domain controls the activity of p63 by an intramolecular mechanism. Mol. Cell. Biol. 22, 8601-8611 (2002).

23. Straub, W. E. et al. The C-terminus of p63 contains multiple regulatory elements with different functions. Cell Death Dis. 1, e5 (2010).

24. Deutsch, G. B. et al. DNA damage in oocytes induces a switch of the quality control factor TAp63a from dimer to tetramer. Cell 144, 566-576 (2011).

25. Suzuki, D., Sahu, R., Leu, N. A. \& Senoo, M. The carboxy-terminus of p63 links cell cycle control and the proliferative potential of epidermal progenitor cells. Development 142, 282-290 (2015).

26. Ranieri, M. et al. Sumoylation and ubiquitylation crosstalk in the control of $\Delta$ Np63a protein stability. Gene $645,34-40$ (2018).

27. Coutandin, D. et al. Quality control in oocytes by p63 is based on a springloaded activation mechanism on the molecular and cellular level. Elife 5, e13909 (2016)

28. Bolcun-Filas, E., Rinaldi, V. D., White, M. E. \& Schimenti, J. C. Reversal of female infertility by Chk2 ablation reveals the oocyte DNA damage checkpoint pathway. Science 343, 533-536 (2014).

29. Tuppi, M. et al. Oocyte DNA damage quality control requires consecutive interplay of CHK2 and CK1 to activate p63. Nat. Struct. Mol. Biol. 25, 261-269 (2018)

30. Michalak, E. M., Villunger, A., Adams, J. M. \& Strasser, A. In several cell types tumour suppressor p53 induces apoptosis largely via Puma but Noxa can contribute. Cell Death Differ. 15, 1019-1029 (2008).

31. Cambiaghi, S., Tadini, G., Barbareschi, M., Menni, S. \& Caputo, R. RappHodgkin syndrome and AEC syndrome: are they the same entity? $\mathrm{Br}$. J. Dermatol. 130, 97-101 (1994).

32. Prontera, P. et al. Cleft palate and ADULT phenotype in a patient with a novel TP63 mutation suggests lumping of EEC/LM/ADULT syndromes into a unique entity: ELA syndrome. Am. J. Med. Genet. 155A, 2746-2749 (2011).

33. Otsuki, Y. et al. Intermediate phenotype between adult syndrome and EEC syndrome caused by R243Q mutation in TP63. Plast. Reconstr. Surg. Glob. Open 4, el185 (2016).

34. Clements, S. E. et al. Rapp-Hodgkin and Hay-Wells ectodermal dysplasia syndromes represent a variable spectrum of the same genetic disorder. $\mathrm{Br}$. J. Dermatol. 163, 624-629 (2010).

35. Celli, J. et al. Heterozygous germline mutations in the p53 homolog p63 are the cause of EEC syndrome. Cell 99, 143-153 (1999).

36. Russo, C. et al. Protein aggregation of the $\mathrm{p} 63$ transcription factor underlies severe skin fragility in AEC syndrome. Proc. Natl Acad. Sci. USA 115, E906-E915 (2018).

37. Guazzarotti, L. et al. Limb-mammary syndrome (LMS) associated with internal female genitalia dysgenesia: A new genotype/phenotype correlation? Am. J. Med. Genet. 146A, 2001-2004 (2008).

38. Holder-Espinasse, M., Martin-Coignard, D., Escande, F. \& Manouvrier-Hanu, S. A new mutation in TP63 is associated with age-related pathology. Eur. J. Hum. Genet. 15, 1115-1120 (2007).

39. Tucker, E. J. et al. Identification of variants in pleiotropic genes causing "isolated" premature ovarian insufficiency: implications for medical practice. Eur. J. Hum. Genet. 26, 1319-1328 (2018).

40. Tucker, E. J. et al. TP63-truncating variants cause isolated premature ovarian insufficiency. Hum. Mutat. 40, 886-892 (2019).

41. Candi, E. et al. Np63 regulates thymic development through enhanced expression of FgfR2 and Jag2. Proc. Natl Acad. Sci. USA 104, 11999-12004 (2007).

42. Sethi, I. et al. A global analysis of the complex landscape of isoforms and regulatory networks of p63 in human cells and tissues. BMC Genomics 16, 584 (2015).

43. Westfall, M. D., Mays, D. J., Sniezek, J. C. \& Pietenpol, J. A. The $\Delta \mathrm{Np63}$ phosphoprotein binds the $\mathrm{p} 21$ and 14-3-3 promoters in vivo and has transcriptional repressor activity that is reduced by Hay-Wells Syndromederived mutations. Mol. Cell. Biol. 23, 2264-2276 (2003).

44. Romano, R. A., Birkaya, B. \& Sinha, S. A functional enhancer of keratin 14 is a direct transcriptional target of $\Delta \mathrm{Np} 63$. J. Invest. Dermatol. 127, 1175-1186 (2007).

45. Cai, B. H., Chao, C. F., Lu, M. H., Lin, H. C. \& Chen, J. Y. A half-site of the p53-binding site on the keratin 14 promoter is specifically activated by p63. J. Biochem. 152, 99-110 (2012).

46. Dotto, J., Pelosi, G. \& Rosai, J. Expression of p63 in thymomas and normal thymus. Am. J. Clin. Pathol. 127, 415-420 (2007). 
47. Senoo, M., Pinto, F., Crum, C. P. \& McKeon, F. p63 is essential for the proliferative potential of stem cells in stratified epithelia. Cell 129, 523-536 (2007).

48. Burnley, P. et al. Role of the p63-FoxN1 regulatory axis in thymic epithelial cell homeostasis during aging. Cell Death Dis. 4, e932 (2013).

49. Rossi, V. et al. LH prevents cisplatin-induced apoptosis in oocytes and preserves female fertility in mouse. Cell Death Differ. 24, 72-82 (2017).

50. Browne, G. et al. Differential altered stability and transcriptional activity of $\Delta$ Np63 mutants in distinct ectodermal dysplasias. J. Cell Sci. 124, 2200-2207 (2011).

51. Wang, P., Yu, J. \& Zhang, L. The nuclear function of p53 is required for PUMA-mediated apoptosis induced by DNA damage. Proc. Natl Acad. Sci. USA 104, 4054-4059 (2007).

52. Schlereth, $\mathrm{K}$. et al. DNA binding cooperativity of p53 modulates the decision between cell-cycle arrest and apoptosis. Mol. Cell 38, 365-368 (2010).

53. Osada, M. et al. p63-specific activation of the BPAG-1e promoter. J. Invest. Dermatol. 125, 52-60 (2005).

54. Chakravarti, D. et al. Induced multipotency in adult keratinocytes through down-regulation of $\triangle \mathrm{Np} 63$ or DGCR8. Proc. Natl Acad. Sci. USA 111, E572-E581 (2014).

55. Pacheco, S. et al. The ATM signaling cascade promotes recombinationdependent pachytene arrest in mouse spermatocytes. PLoS Genet. 11, E1005017 (2015)

56. Marcet-Ortega, M. et al. p53 and TAp63 participate in the recombinationdependent pachytene arrest in mouse spermatocytes. PLoS Genet. 13, 1006845 (2017).

57. Ghioni, P. et al. The protein stability and transcriptional activity of p63a are regulated by SUMO-1 conjugation. Cell Cycle 4, 183-190 (2005).

58. Rossi, M. et al. The E3 ubiquitin ligase Itch controls the protein stability of p63. Proc. Natl Acad. Sci. USA 103, 12753-12758 (2006).

59. Schwenk, F., Baron, U. \& Rajewsky, K. A cre-transgenic mouse strain for the ubiquitous deletion of loxP-flanked gene segments including deletion in germ cells. Nucleic Acids Res. 23, 5080-5081 (1995).

60. Cairns, L. A. et al. Kit regulatory elements required for expression in developing hematopoietic and germ cell lineages. Blood 102, 3954-3962 (2003).

61. Osada, M. et al. Differential recognition of response elements determines target gene specificity for p53 and p63. Mol. Cell. Biol. 25, 6077-6089 (2005).

62. Yu, J., Zhang, L., Hwang, P. M., Kinzler, K. W. \& Vogelstein, B. PUMA induces the rapid apoptosis of colorectal cancer cells. Mol. Cell 7, 673-682 (2001).

\section{Acknowledgements}

This work has been mainly supported by AIRC Grants (IG-22206 to E.C.) and Ministry of Health and Fondazione Luigi Maria Monti IDI-RCCS (RC to E.C.). The work was also partially supported by AIRC (IG\#20473, 2017-2022; Start-up ID23219, 2019-2024). V.D acknowledges support by DFG (DO 545/20-1).

\section{Author contributions}

A.M.L., V.R., S.O., A.S., C.O., M.T. and A.C. performed the experiments. A.M.L., V.R and A.S. characterized mouse phenotype (skin, thymus, ovary), S.O., C.O. and M.T. performed transcription activity assays and oligomerization studies. E.C., A.M.L., F.G.K. and V.D. designed the research; A.M.L., V.R., S.O., A.S., C.O., A.C., F.G.K., M.A.P., I.A., H.V., M.D.F. and G.M. analysed the data; E.C. and V.D. wrote the paper; all the authors read the paper and made comments.

\section{Competing interests}

The authors declare no competing interests.

\section{Additional information}

Supplementary information is available for this paper at https://doi.org/10.1038/s41467020-20669-0.

Correspondence and requests for materials should be addressed to G.M. or E.C.

Peer review information Nature Communications thanks Karla Hutt and the other, anonymous, reviewer(s) for their contribution to the peer review of this work. Peer reviewer reports are available.

Reprints and permission information is available at http://www.nature.com/reprints

Publisher's note Springer Nature remains neutral with regard to jurisdictional claims in published maps and institutional affiliations.

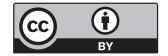

Open Access This article is licensed under a Creative Commons Attribution 4.0 International License, which permits use, sharing, adaptation, distribution and reproduction in any medium or format, as long as you give appropriate credit to the original author(s) and the source, provide a link to the Creative Commons license, and indicate if changes were made. The images or other third party material in this article are included in the article's Creative Commons license, unless indicated otherwise in a credit line to the material. If material is not included in the article's Creative Commons license and your intended use is not permitted by statutory regulation or exceeds the permitted use, you will need to obtain permission directly from the copyright holder. To view a copy of this license, visit http://creativecommons.org/ licenses/by/4.0/.

(C) The Author(s) 2021 